\title{
Anyonic Defect Braiding and Spontaneous Chiral Symmetry Breaking in Dihedral Liquid Crystals
}

\author{
Alexander Mietke $\odot^{*}$ and Jörn Dunkelø $\oplus^{\dagger}$ \\ Department of Mathematics, Massachusetts Institute of Technology, \\ 77 Massachusetts Avenue, Cambridge, Massachusetts 02139-4307, USA
}

(Received 10 November 2020; revised 8 December 2021; accepted 9 December 2021; published 9 February 2022)

\begin{abstract}
Dihedral (" $k$-atic") liquid crystals (DLCs) are assemblies of microscopic constituent particles that exhibit $k$-fold discrete rotational and reflection symmetries. Generalizing the half-integer defects in nematic liquid crystals, two-dimensional $k$-atic DLCs can host point defects of fractional topological charge $\pm m / k$. Starting from a generic microscopic model, we derive a unified hydrodynamic description of DLCs with aligning or antialigning short-range interactions in terms of Ginzburg-Landau and Landau-BrazovskiiSwift-Hohenberg theories for a universal complex order-parameter field. Building on this framework, we demonstrate in particle simulations how adiabatic braiding protocols, implemented through suitable boundary conditions, can emulate anyonic exchange behavior in a classical system. Analytic solutions and simulations of the mean-field theory further predict a novel spontaneous chiral symmetry-breaking transition in antialigning DLCs, in quantitative agreement with the patterns observed in particle simulations.
\end{abstract}

DOI: $10.1103 /$ PhysRevX.12.011027

Subject Areas: Soft Matter

\section{INTRODUCTION}

Microscopic particle symmetries fundamentally determine the macroscopic order and dynamics of liquid and crystalline phases of matter [1,2]. Recent technological and experimental progress [3-5] has enabled unprecedented precise control over the fabrication and assembly of nanoparticles [6,7] and polyhedral colloids [8-14] with tunable interactions $[10,15-17]$. These advances have led to a renewed theoretical and computational interest in $k$-atic dihedral liquid crystals (DLCs) with discrete $k$-fold rotational and reflection symmetries [18-28]. Going beyond the widely investigated polar $(k=1)$ and nematic $(k=2)$ liquid crystals [1], recent studies have shown that assemblies of triatic [22] $(k=3)$ and higher-order polygonal [21] objects $(k \geq 3)$ can exhibit striking symmetry-breaking phenomena. Furthermore, thanks to seminal work by de Gennes [29], Halperin and Lubensky [30], and others [31-36], it is well known that the phenomenological description of 2D liquid crystals shares interesting mathematical similarities [37] with superconductors. Despite their fundamental microscopic differences, both classes of

\footnotetext{
*amietke@mit.edu

†unkel@mit.edu
}

Published by the American Physical Society under the terms of the Creative Commons Attribution 4.0 International license. Further distribution of this work must maintain attribution to the author(s) and the published article's title, journal citation, and DOI. systems can, at the mean-field level, be described by a complex field $\Psi(t, \mathbf{r})=|\Psi| e^{i \phi}$ whose magnitude $|\Psi|$ and phase $\phi(t, \mathbf{r})$ encode local order.

A remarkable characteristic of two-dimensional (2D) DLCs is their ability to host point defects of fractional topological charge [22,34], similar to anyonic quasiparticle excitations in 2D quantum matter [38,39]. One of the defining features of anyonic excitations is the behavior of their wave function under pair exchange: When two identical anyons with initial positions $\mathbf{r}_{1}$ and $\mathbf{r}_{2}$ are braided counterclockwise around each other, their complex wave function $\psi$ changes according to $\psi\left(\mathbf{r}_{2}, \mathbf{r}_{1}\right)=e^{i 2 \pi / p} \psi\left(\mathbf{r}_{1}, \mathbf{r}_{2}\right)$, where $p=1,2, \ldots$. In other words, anyonic wave functions acquire a phase $\theta=2 \pi / p$ under particle exchange, which we refer to as an anyonic exchange symmetry in this work; bosons and fermions correspond to the special cases $p=1$ and $p=2$, respectively. First predicted [40] in 1977 and named [38] in 1982, anyons played an important role in the theoretical explanation of the fractional quantized Hall effect [41-43]. Nearly four decades later, two recent experimental studies $[44,45]$ reported the first direct evidence for anyonic quantum statistics. The long search for physically realizable anyonic excitations and their intense theoretical exploration has, in part, been driven by the prospect of performing anyonbased topological quantum computing [46,47]. The mathematical parallels between the mean-field descriptions of 2D liquid crystals and 2D quantum systems therefore raise the interesting question of whether one can emulate anyonic exchange symmetries in suitably designed liquid crystal systems. 
Here, we show that it is indeed possible to create, stabilize, and manipulate pairs of identical fractional defects $\left(\mathbf{r}_{1}, \mathbf{r}_{2}\right)$ in $k$-atic DLCs, such that a braiding exchange $\left(\mathbf{r}_{1}, \mathbf{r}_{2}\right) \rightarrow$ $\left(\mathbf{r}_{2}, \mathbf{r}_{1}\right)$ gives rise to anyonic exchange symmetries in the local polar particle orientations, and is accompanied by the accumulation of a globally constant phase difference in the $k$ atic order-parameter field $\Psi_{k}$. Specifically, we find that only two essential ingredients are needed to realize these effects: a short-range $k$-fold symmetric particle-particle interaction that can be aligning or antialigning, and a tunable boundary anchoring that exploits defect topology and energetics to control the distance between defects and domain boundary through anchoring inhomogeneities. Dynamic control of the defect positions through modulation of the boundary anchoring enables defect braiding and, in particular, provides control over branch-cut singularities in a DLC. These singularities inherit the fractional properties of DLC defects and, in the course of a braiding operation, carve out subdomains on which an anyonic exchange symmetry emerges. We demonstrate this phenomenon in simulations of a generic $k$-atic $X Y$-type particle model, which can be analytically coarse grained to obtain a hydrodynamic meanfield description. The resulting hydrodynamic continuum model, which encompasses Landau-Brazovskii systems [48], permits analytic solutions that predict a novel chiral symmetry-breaking transition in antialigning $k$-atic DLCs, characterized by the spontaneous formation of stable, braidable spiral patterns in the phase angle fields. We confirm this symmetry-breaking transition in explicit particle simulations and generally observe remarkably close agreement between mean-field predictions and particle dynamics. The generic nature of the underlying particle interaction model suggests that experimental realizations of the theoretical predictions presented below could be implemented in a variety of systems. Potential candidate systems that may serve as starting points to develop such realizations range from threefold symmetric molecules (e.g., 1,3,5-trichlorobenzene as proposed in Ref. [22]) or DNA-origami structures [49-51] to $k$-atic colloidal platelets [8,14] and polyhedral [4], magnetic [52], or DNA-coated colloids [9,53] (see also Fig. 1 in Ref. [28]).

\section{MEAN-FIELD DESCRIPTION OF 2D DLCs WITH ALIGNING AND ANTIALIGNING INTERACTIONS}

Generalizations of polar and nematic liquid crystals to higher-order symmetry groups are often called $k$-atics [34], where the integer $k$ counts the discrete rotational symmetries of the constituent particles [Figs. 1(a) and 1(b)]. (a)

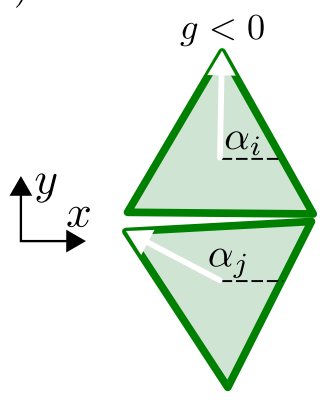

(d) $\quad k=3$

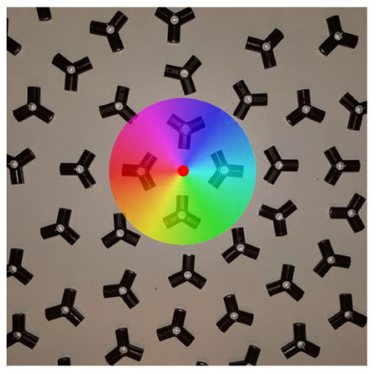

(b)

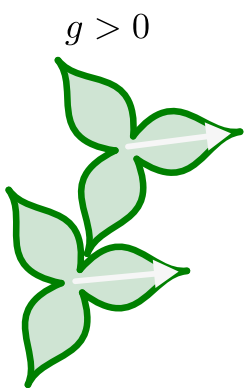

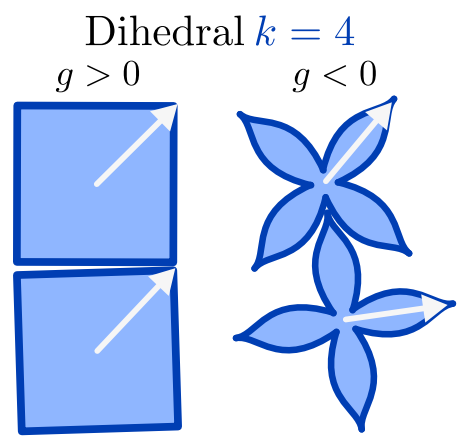

(c) Cyclic/Chiral

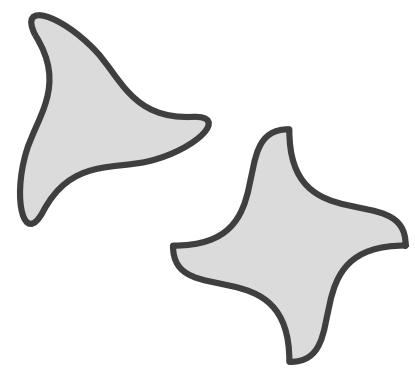

(e) $k=3 \quad m=1$
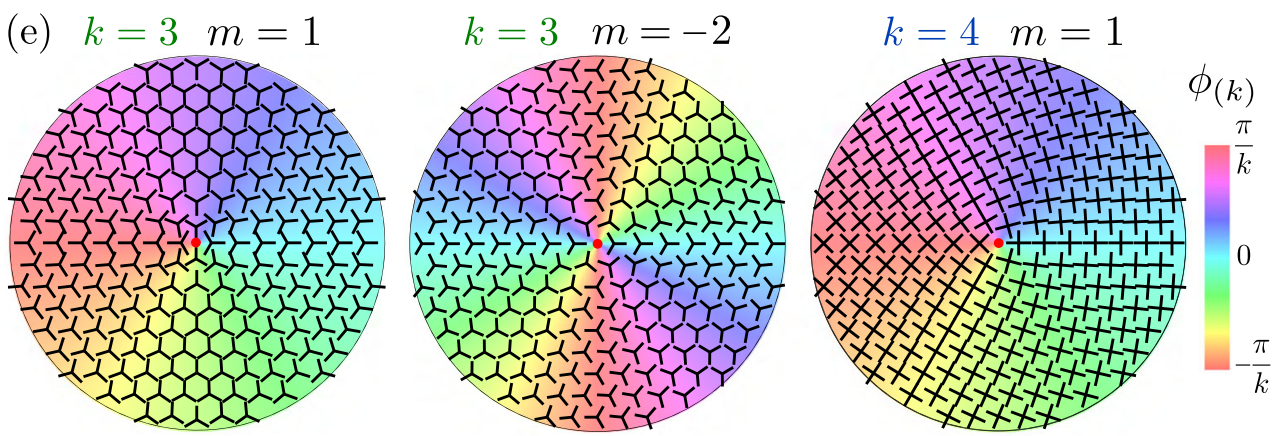

FIG. 1. Illustration of DLC particles, $k$-atic order, and point defects carrying fractional topological charges $m / k$. (a,b) Examples of 3 -atic $(k=3)$ and 4-atic $(k=4)$ dihedral particles with aligning $(g>0)$ and antialigning $(g<0)$ interactions. Director vectors are shown as white arrows. (c) Examples of chiral non-DLC particles that exhibit $k$-fold rotational (cyclic) symmetry but lack reflection symmetry. The present study focuses exclusively on dihedral particles as in panels (a) and (b). (d) Realization of a $+1 / 3$ defect in the collection of three-legged symmetric LEGO connectors with mutually repulsive magnetic interactions. For additional experimental realizations of $k$-atic liquid crystals in colloidal suspensions, see, for example, Refs. [8,14]. (e) From left to right: $k$-atic phase angle fields $\phi_{(k)}$ for point defects of topological charge $+1 / 3$ in a 3 -atic $(k=3, m=1)$, charge $-2 / 3$ in a 3 -atic $(k=3, m=-2)$, and charge $+1 / 4$ in a 4 -atic field $(k=4, m=1)$ with local $k$-atic directors overlaid (see also Fig. 8 for additional examples). 
Here, we focus on systems of particles that have both $k$-fold discrete rotational and reflection symmetry [Figs. 1(a) and 1(b)]. This set of symmetry transformations defines the dihedral group $D_{k}$, which maps a regular polygon with $k$ corners onto itself. Accordingly, we refer to such systems generically as DLCs. Note that the invariance under reflections excludes chiral particles, which can still have discrete rotational (cyclic) symmetry [Fig. 1(c)]. Depending on whether $k$ is even or odd, and whether the effective particle shape is convex or concave, DLCs can have aligning or antialigning interactions [Figs. 1(a) and 1(b)]. Here, "shape" can be understood in a broader sense as the angular symmetry of the particle's pair-interaction potential, for which we provide a concrete example in Sec. III. As we show in detail below, monodisperse DLCs with aligning and antialigning interactions can be described by a single, universal, meanfield equation. Before delving into a more technical discussion, it is instructive to anticipate the structure of the resulting mean-field equations and their relations to anyonic exchange symmetries.

\section{A. Universal mean-field equation}

In the limit of a constant particle density, a unified mean-field description of 2D monodisperse DLCs can be given in terms of a complex-valued scalar orderparameter field $\Psi_{k}(t, \mathbf{r})$ that is governed by the relaxation dynamics

$$
\tau \partial_{t} \Psi_{k}=-\left(A+B\left|\Psi_{k}\right|^{2}\right) \Psi_{k}+\mathcal{L}\left(\nabla^{2}\right) \Psi_{k}=-\frac{\delta \mathcal{E}_{k}}{\delta \Psi_{k}^{*}} .
$$

Here, $\mathcal{L}\left(\nabla^{2}\right)$ denotes a linear differential operator, and $\mathcal{E}_{k}$ is the corresponding energy functional. The subscript $k$ indicates the $k$-fold symmetry, and $\tau$ is a relaxation timescale that can be computed from the microscopic particle dynamics. The magnitude $\left|\Psi_{k}\right|$ characterizes the degree of local order (alignment) of the director unit vectors [white arrows in Figs. 1(a) and 1(b)], and the $k$-atic phase angle of $\Psi_{k}$ is their mean orientation.

As shown in detail below, the real parameters $A$ and $B$ depend on the particle symmetry and interaction strength. For aligning interactions, the operator $\mathcal{L}$ in Eq. (1) reduces to a Laplacian $\mathcal{L}=L^{2} \nabla^{2}$; in this case, Eq. (1) corresponds a "real" Ginzburg-Landau (GL) equation [54] with an effective diffusion constant $D=L^{2} / \tau$ (Sec. IV). For antialigning interactions, $\mathcal{L}$ will take the form of a pattern-forming Swift-Hohenberg-type (SH) [55] operator $\mathcal{L}=-L_{1}^{2} \nabla^{2}-L_{2}^{4}\left(\nabla^{2}\right)^{2}$ (Sec. V).

Conceptually, Eq. (1) formalizes the mathematical correspondence between the mean-field descriptions of aligning DLCs and quantum fluids. If $\mathcal{L}$ is proportional to the Laplacian, the energy $\mathcal{E}_{k}$ in Eq. (1) takes the form [34] (Appendix B 1)

$$
\mathcal{E}_{k}=\int d^{2} r\left(A\left|\Psi_{k}\right|^{2}+\frac{B}{2}\left|\Psi_{k}\right|^{4}+L^{2}\left|\nabla \Psi_{k}\right|^{2}\right)
$$

Then, for $k=1$ and $\tau=i$ in Eq. (1), one recovers the Gross-Pitaevskii equation [56,57] describing Bose-Einstein condensates. Thus, 2D aligning DLCs and quantum fluids can be considered energetically equivalent at the mean-field level while differing by the fact that the former have dissipative dynamics whereas the latter have conservative dynamics. Similar mean-field correspondences played a historically important role for the understanding of smectic liquid crystal phases by their analogy with superconductors $[29,30,33]$.

For the SH-type mean-field theory of DLCs with antialigning interactions, $\mathcal{E}_{k}$ in Eq. (1) corresponds to a Landau-Brazovskii (LB) energy [48] (Appendix C 1). The LB energy functional generally captures the mean-field dynamics of systems with competing microscopic interactions, such as diblock copolymere suspensions [58,59] or microemulsions $[60,61]$, and it plays a key role in explaining how fluctuations affect the properties of order-disorder phase transitions [48,62-64].

In the context of our present study, Eq. (1) provides the basis for realizing classical counterparts of anyon exchange symmetries. More specifically, we see below that Eq. (1) accurately describes the formation, stability, and decay (Figs. 2 and 5), and the braiding (Figs. 4 and 7) of fractional topological defects as observed in particle simulations of generic $X Y$-type microscopic DLC models. When appropriately braided around each other, these fractional topological defects mimic the behavior of anyonic quasiparticle excitations by acquiring a phase shift in the complex orderparameter field.

\section{B. Fractional topological charges}

To characterize the orientational order of DLCs, one can express the complex order-parameter field in the polar form (Appendix B 1)

$$
\Psi_{k}=\left|\Psi_{k}\right| e^{i k \phi_{(k)}} .
$$

The magnitude field $\left|\Psi_{k}\right|$ measures the strength of the local $k$-atic order, and the phase angle field

$$
\phi_{(k)}=\frac{\arg \Psi_{k}}{k}
$$

indicates the mean $k$-atic director orientation. The widely studied polar and nematic liquid crystals correspond to $k=1$ and $k=2$, with microscopic constituents symmetric under rotations of $2 \pi$ or $\pi$, respectively.

The phase field $\phi_{(k)}$ of a $k$-fold symmetric DLC can host commensurate fractional point defects (Appendix B 2). The net topological defect charge $q_{\mathrm{d}}$ enclosed by a positively oriented curve $\mathcal{C}$ is obtained as 

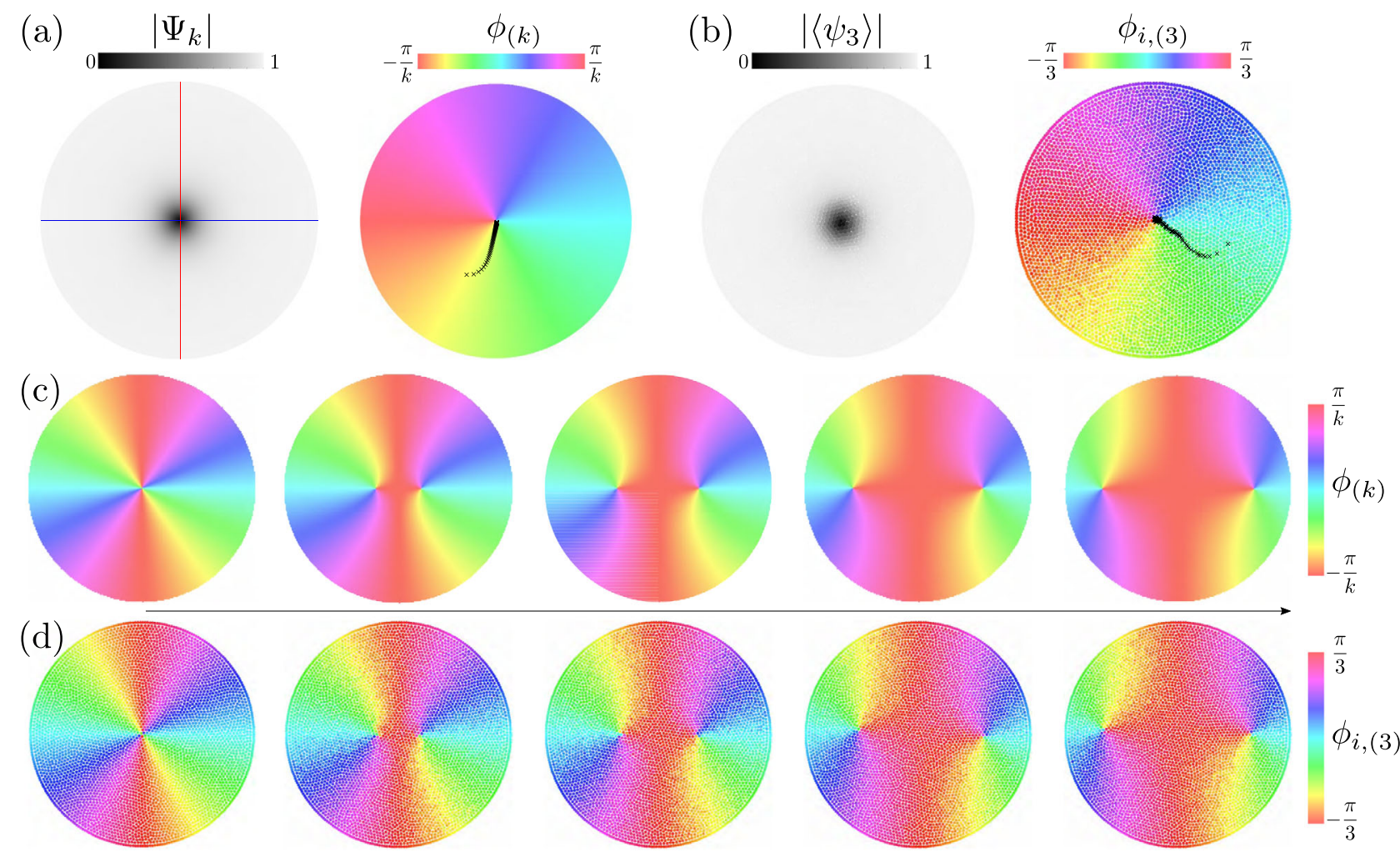

FIG. 2. Mean-field theory correctly predicts defect formation, stability, and decay as seen in the particle model with aligning interactions $(g>0)$ on a unit disk. (a) Stationary $1 / k$-defect solution emerging from random initial conditions in the mean-field equation (1) with $\mathcal{L}=L^{2} \nabla^{2}, A=-1, B=1, L=0.08$, for boundary anchoring [Eq. (14)] with $m=1$ and $\gamma_{k}=\theta$. The lines Re $\Psi_{k}=0$ (red) and $\operatorname{Im} \Psi_{k}=0$ (blue) indicate how the boundary anchoring induces a point defect with $\left|\Psi_{k}\right|=0$ inside the unit disk. The defect trajectory (black) shows that the defect appears at a random position in the bulk and subsequently moves towards the center of the disk (Supplemental Movie 1 [89]). (b) Steady-state 1/3 defect emerging from random initial conditions in particle simulations of Eq. (6) with $k=3$, using an equivalent orientational boundary-anchoring equation (17) with $\gamma_{k}=\theta$. Here, $\left\langle\psi_{3}\right\rangle$ denotes the time average of the local 3 -atic order parameter $\psi_{3}=\sum_{j \in \mathcal{N}_{i}} \exp \left(3 i \alpha_{j}\right) /\left|\mathcal{N}_{i}\right|$ at the steady state, and $\phi_{i,(k)}=\arg \left(e^{i k \alpha_{i}}\right) / k$ is the instantaneous $k$-atic director orientation. In agreement with the mean-field prediction, the defect trajectory (black) shows that a phase defect forms at a random position in the bulk and moves stochastically towards the center of the disk (Supplemental Movie 1 [89]). (c,d) For profiles $\gamma_{k}=\theta$ and $m=2$ in boundary-anchoring equations (14) and (17), a $2 / k$ defect decays into two $1 / k$ defects in both the mean-field simulations (c) and 3-atic particle simulations (d) (Supplemental Movie 2 [89]). Snapshots show instantaneous phase fields at $t / \tau \in$ $\{0,7.5,15,37.5,150\}$ [Eq. (1); (c)] and $t / \bar{\tau} \in\{0,18,36,80,320\}$ [particle model; (d)]. Simulations of Eq. (6) used 4000 nonanchored bulk particles, 900 anchored boundary particles, and parameters $D_{r}=1$ (rotational diffusion chosen as the characteristic timescale of particle simulations), $R_{\alpha}=0.2$ (interaction radius), and $g=0.25$ (interaction strength).

$$
q_{\mathrm{d}}=\frac{1}{2 \pi} \oint_{\mathcal{C}} d \mathbf{l} \cdot \nabla \phi_{(k)}=\frac{m}{k}
$$

where $m$ can be any integer. Examples of defect states in $k$-atic DLCs are illustrated in Figs. 1(d), 1(e), and 8. The "experimental" realization of the $+1 / 3$ defect in Fig. 1(d) was assembled from 3-atic LEGO toy elements that are invariant under $2 \pi / 3$ rotations and corresponding reflections, and carry repulsive magnetic dipoles in each of their legs. Owing to the discrete symmetry of each microscopic element, only a rotation by $2 \pi / k$ is required before a particle returns to its initial configuration. If particle orientations along the integration contour complete $|m|$ counterclockwise rotations in total, then the defect charge is positive with $m>0$ in Eq. (5), whereas $m<0$ signals the completion of $|m|$ clockwise $2 \pi / k$ rotations along the curve $\mathcal{C}$.

\section{MICROSCOPIC DLC MODEL}

A priori, it is not clear how well a mean-field model can capture the behavior of a specific microscopic DLC system. To validate predictions obtained from Eq. (1), we compare them against simulations of a generic microscopic DLC model that can realize both aligning and antialigning interactions. Specifically, we consider an $X Y$-type model [65] describing particles with local orientation angles $\alpha_{i}(t) \in[0,2 \pi)$ that interact according to the overdamped dynamics 


$$
\frac{d \alpha_{i}}{d t}=\frac{g}{\pi R_{\alpha}^{2}} \sum_{j \in \mathcal{N}_{i}} \sin \left[k\left(\alpha_{j}-\alpha_{i}\right)\right]+\sqrt{2 D_{r}} \xi_{i}(t) .
$$

Equation (6) is invariant under rotations $\alpha_{i} \rightarrow \alpha_{i}+2 \pi / k$ and corresponding reflections along the symmetry axis of the particles. The parameter $g$ sets the effective interaction strength between particle $i$ and particles $j$ in a neighborhood $\mathcal{N}_{i}$ of radius $R_{\alpha}$. For $g>0$, the $k$-atic directors of nearby particles tend to align, whereas $g<0$ favors antialignment [Figs. 1(a) and 1(b)]. The Gaussian white noise $\xi_{i}(t)$ has zero mean and satisfies $\left\langle\xi_{i}(t) \xi_{j}\left(t^{\prime}\right)\right\rangle=\delta_{i j} \delta\left(t-t^{\prime}\right)$, and $D_{r}$ is the rotational diffusion constant. In all simulations presented below, particles were randomly placed inside a circular disk domain and given time to redistribute homogeneously through isotropic short-range repulsion (Appendix D 4). Thereafter, the particle positions were held fixed, and the angular dynamics Eq. (6) was turned on. This model may thus be interpreted as a generalized classical $X Y$ model on a densely packed random lattice [66].

\section{A. Discrete $k$-scaling invariance}

In agreement with mean-field predictions, simulations for particles with $k$-fold symmetry show topological defects of fractional charges that are integer multiples of $1 / k$ (Fig. 2). We note, however, that for any value of $k$, the dynamics given in Eq. (6) can be mapped onto an equivalent polar model with $k=1$, by defining rescaled director angles $\alpha_{i}^{\prime}=k \alpha_{i}$, a rescaled alignment strength $g^{\prime}=g k$, and a rescaled rotational diffusion $D_{r}^{\prime}=D_{r} k^{2}$. The fact that Eq. (6) can be rescaled in this form essentially explains why the DLC particle models with different $k$ can be described by the same mean-field equation (1). Although, as we discuss next, the coefficients in Eq. (1) depend on $k$, the structure of the mean-field equation remains preserved for particles with different dihedral symmetries.

\section{B. Mean-field parameters}

To show how Eq. (1) can be derived from the microscopic model in Eq. (6), we generalize standard coarse-graining procedures [67-69] to the case of $k$-atic particle interactions with a finite spatial range [70-72] (Appendix A). To this end, we decompose the one-particle probability density function of the $N$-particle system, which is defined by the Gaussian white-noise average

$$
f(\alpha, \mathbf{r}, t)=\sum_{i=1}^{N}\left\langle\delta\left[\alpha-\alpha_{i}(t)\right] \delta\left(\mathbf{r}-\mathbf{r}_{i}\right)\right\rangle,
$$

into its angular moments

$$
f_{n}(\mathbf{r}, t)=\int_{0}^{2 \pi} d \alpha f(\alpha, \mathbf{r}, t) e^{i n \alpha}
$$

The mode $f_{0}$ represents the particle number density $\rho$ of the system, which, in our case, is homogeneous and fixed. Accordingly, we define normalized and dimensionless modes by

$$
\psi_{n}=\frac{f_{n}}{\rho} .
$$

Equations (6) and (8) yield an infinite hierarchy of dynamic equations for the complex modes $\psi_{n}$ (Appendix A). Despite being nonlinear, these equations decouple modes with $n=j k$ for integers $j \neq 0$ from all modes with $n \neq j k$, which can be understood as a consequence of the $k$-rescaling property of the microscopic model in Eq. (6). In the limit of a vanishing interaction radius $R_{\alpha} \rightarrow 0$, one then finds the spatially homogeneous dynamics (Appendix A 1)

$$
\bar{\tau} \partial_{t} \psi_{k}=-\left(\bar{A}+\bar{B}\left|\psi_{k}\right|^{2}\right) \psi_{k}=: h_{k},
$$

with characteristic relaxation timescale $\bar{\tau}=2 /(|g| k \rho)$ and parameters

$$
\bar{A}=-\operatorname{sgn}(g)\left(1-\frac{2 D_{r} k}{g \rho}\right), \quad \bar{B}=\frac{|g| \rho}{4 D_{r} k} .
$$

By comparing Eqs. (10) and (11) with Eq. (1), we can identify $\psi_{k} \simeq \Psi_{k}, \bar{\tau} \simeq \tau, \bar{A} \simeq A$, and $\bar{B} \simeq B$, indicating that the particle dynamics (6) indeed provides a microscopic realization of the mean-field theory (1). Below, we extend Eq. (10) to short-range interactions with $R_{\alpha}>0$, which leads to leading-order corrections in the form of linear operators $\mathcal{L}\left(\nabla^{2}\right)$ as indicated in Eq. (1).

Beforehand, we note that Eq. (10) generalizes the corresponding result [68] for polar systems $(k=1)$ to arbitrary $k$-atic systems. A change in the sign of the coefficient $\bar{A}$ signals the spontaneous emergence of homogeneous $k$-atic order due to a linear instability of Eq. (10) if $g>g^{*}:=2 k D_{r} / \rho>0$, corresponding to an instability at wave vector $\mathbf{q}=0$. This also implies that for antialigning interactions with $g<0$, the disordered state is linearly stable in the limit of pointwise interactions.

In the remainder of this paper, we consider the experimentally relevant case of systems with finite interaction range $R_{\alpha}>0$. By comparing the mean-field predictions of Eq. (1) with the quantitatively mapped microscopic models described by Eq. (6), we demonstrate the controlled manipulation of fractional defects through boundary anchoring (Sec. IV) and the spontaneous formation of chiral textures (Sec. V).

\section{DEFECT BRAIDING AND ANYONIC EXCHANGE SYMMETRIES IN ALIGNING DLCs $(g>0)$}

We first show that for aligning short-range interactions in the particle model in Eq. (6), the mean-field description (1) takes the form of a "real" GL equation. Thereafter, 
we identify boundary-anchoring conditions that will allow us to position and manipulate fractional defects in both mean-field and particle simulations. We then apply this framework to braid two identical point defects, which gives rise to an emergent anyonic exchange symmetry in the particle model. Finally, a protocol of boundary-anchoring modulations is proposed for which a global $k$-atic phase change arises in complex order-parameter fields described by both mean-field and particle simulations.

\section{A. GL mean-field theory for aligning $\boldsymbol{k}$-atics}

Assuming aligning interactions $[g>0$; Figs. 1(a) and 1(b)] and an isotropic interaction neighborhood in the particle model Eq. (6), the spatiotemporal $k$-atic mode dynamics can be approximated by (Appendix A 2)

$$
\bar{\tau} \partial_{t} \psi_{k}(\mathbf{r}, t) \approx h_{k}+\frac{R_{\alpha}^{2}}{8} \nabla^{2} \psi_{k}(\mathbf{r}, t),
$$

with the homogeneous part $h_{k}$ given in Eq. (10). Note that the effective diffusion constant $\bar{D}=R_{\alpha}^{2} /(8 \bar{\tau})$ is not the result of actual particle diffusion but instead arises from the finiterange interactions between particles at fixed positions.

Equation (12) shows that the mean-field description of aligning DLC particle systems is given by Eq. (1) with

$$
\mathcal{L}=L^{2} \nabla^{2}, \quad L \simeq \frac{R_{\alpha}}{\sqrt{8}}
$$

corresponding to a "real" GL equation [54] for the $k$-atic order parameter $\Psi_{k}$. The correlation length $L$ is related to an effective bending rigidity that penalizes deviations of the $k$-atic director field from a homogeneously aligned state $[1,73]$ (Appendix B 1). We now exploit this feature to generate and position topological defects through appropriate boundary conditions.

\section{B. Defect positioning through boundary anchoring}

To illustrate how the total topological charge and the positioning of $k$-atic defects can be controlled, we consider numerical solutions of the GL equation (1) on a unit disk domain $S$ with boundary anchoring

$$
\left.\Psi_{k}\right|_{\partial S}=e^{i m \gamma_{k}} .
$$

Through a prescribed anchoring profile $\gamma_{k}(\theta)$, Eq. (14) fixes the orientation of the $k$-atic director along the boundary $\partial S$ to

$$
\phi_{(k)}=\frac{1}{k} \arg \left(e^{i m \gamma_{k}}\right)
$$

The examples discussed in the remainder of this paper are based on a monotonically increasing profile $\gamma_{k}(\theta)$ with $\gamma_{k}(2 \pi)-\gamma_{k}(0)=2 \pi$, for which the boundary condition (14) imposes a total topological charge $m / k$ in the disk.

The simplest nontrivial anchoring condition (14), corresponding to a topological net charge of $1 / k$ in the disk, is $m=1$ and $\gamma_{k}=\theta$. In this case, the GL relaxation dynamics favors the formation of a stationary $1 / k$ defect at the center of the disk [Fig. 2(a); Supplemental Movie 1 [89]]. Although this could have been expected on symmetry grounds, it is effectively a consequence of the director field's bending rigidity mediated by $L$ : A central position reduces the distortions of the director field around the defect to a single, monotonous winding that is minimally necessary to be compatible with the boundary conditions.

When imposing $m=2$ in Eq. (14), the net topological charge in the disk is $2 / k$. For suitable initial conditions, this charge may first be concentrated in a single defect, which then splits into a pair of $1 / k$ defects with lower energy [Fig. 2(c); Supplemental Movie 2 [89] ]. The final steady state reflects the fact that it is energetically favorable to distribute the director-field distortions around two $1 / k$ defects, instead of winding the director field symmetrically but with twice the rate around a single $2 / k$ defect. Similarly for $m>2$ and $\gamma_{k}=\theta$, transient higher-charge defects decay into $m$ single $1 / k$ defects that eventually settle into symmetric low-energy configurations [Figs. 10(a) and 10(b)].

To test if these predictions can indeed be reproduced in the particle model, we simulate Eqs. (6) on a unit disk [Figs. 2(b) and 2(d)], where a particle orientation $\alpha_{i}$ corresponds to a $k$-atic director orientation

$$
\phi_{i,(k)}=\frac{1}{k} \arg \left(e^{i k \alpha_{i}}\right) .
$$

Comparing this with Eq. (15), the mean-field boundary condition (14) can be matched by fixing the orientations of particles at the boundary to

$$
\left.\alpha_{i}\right|_{\partial S}=(m / k) \gamma_{k}
$$

Note that particle orientations $\alpha_{i}$ and $k$-atic director orientations $\phi_{i,(k)}$ [Eq. (6)] are two distinct observables. In particular, $\phi_{i, k)} \in(-\pi / k, \pi / k]$ can be unambiguously determined for dihedral shapes with $k$ sides, while measuring a corresponding particle orientation $\alpha_{i} \in[0,2 \pi)$ requires an additional polar feature as indicated by the white arrows in Fig. 1. For example, in $k$-atic colloidal systems $[8,14]$, it would suffice to mark one corner of each platelet for tracking purposes.

Using the same monotonous boundary anchoring $\gamma_{k}=\theta$, we find that the resulting particle simulations [Figs. 2(b), 2(d), 10(a), and 10(b)] agree well with the GL theory. For example, for $k=3$ and $m=1$, a single $1 / 3$ defect forms and moves to the center of the domain [Fig. 2(b); Supplemental Movie 1 [89] ], whereas for $k=3$ and $m=2$, an initially 
created $2 / 3$ defect splits into two $1 / 3$ defects that move symmetrically away from each other until they reach a symmetric steady-state position [Fig. 2(d); Supplemental Movie 2 [89] ]. In both cases, the final steady-state textures confirm the GL mean-field prediction.

The above examples illustrate how topological defects take equilibrium positions that effectively minimize director winding gradients around them. It follows that for a constant azimuthal anchor-winding gradient $\left(\partial_{\theta} \gamma_{k}=\right.$ const $)$ at the disk boundary, the equilibrium positions of defect pairs are degenerate with respect to rotations around the disk center. In turn, structured anchoring enables a targeted defect positioning. We demonstrate this useful fact by controlling the orientation of the axis connecting a $1 / k$-defect pair. To this end, we consider Eq. (17) with $m=2$ and the anchoring profile

$$
\gamma_{k}(\theta)=\theta-\theta_{0}+a \sin \left[2\left(\theta-\theta_{\mathrm{a}}\right)\right],
$$

where $a$ sets the strength of the defect anchoring and $|a|<1 / 2$ ensures that $\gamma_{k}(\theta)$ is monotonic. This choice of $\gamma_{k}(\theta)$ is motivated as follows (consider $\theta_{0}=0$, for simplicity): For $\theta_{\mathrm{a}}=0$ and $a>0$, the azimuthal anchor-winding gradient $\partial_{\theta} \gamma$ becomes maximal at $\theta=0, \pi$ and minimal at $\theta=\pi / 2,3 \pi / 2$. It is therefore energetically favorable for topological defects to be closer to the boundary at $\theta=0, \pi$ than at $\theta=\pi / 2,3 \pi / 2$. Consequently, the axis of a defect pair aligns with the $x$ axis in this case [Fig. 3(a), $t=0$ ]. An analogous reasoning for arbitrary $\theta_{\mathrm{a}}$ implies that the boundary condition (18) orients defect pairs along the axis $\left(\cos \theta_{\mathrm{a}}, \sin \theta_{\mathrm{a}}\right)$ when $a>0$. In the next subsection, we use a dynamic generalization of the anchoring profile in Eq. (18) to realize defect braiding protocols in both continuum and particle simulations.

\section{Braiding through boundary modulation}

We now demonstrate how one can implement a braiding protocol that mimics the properties of anyonic states
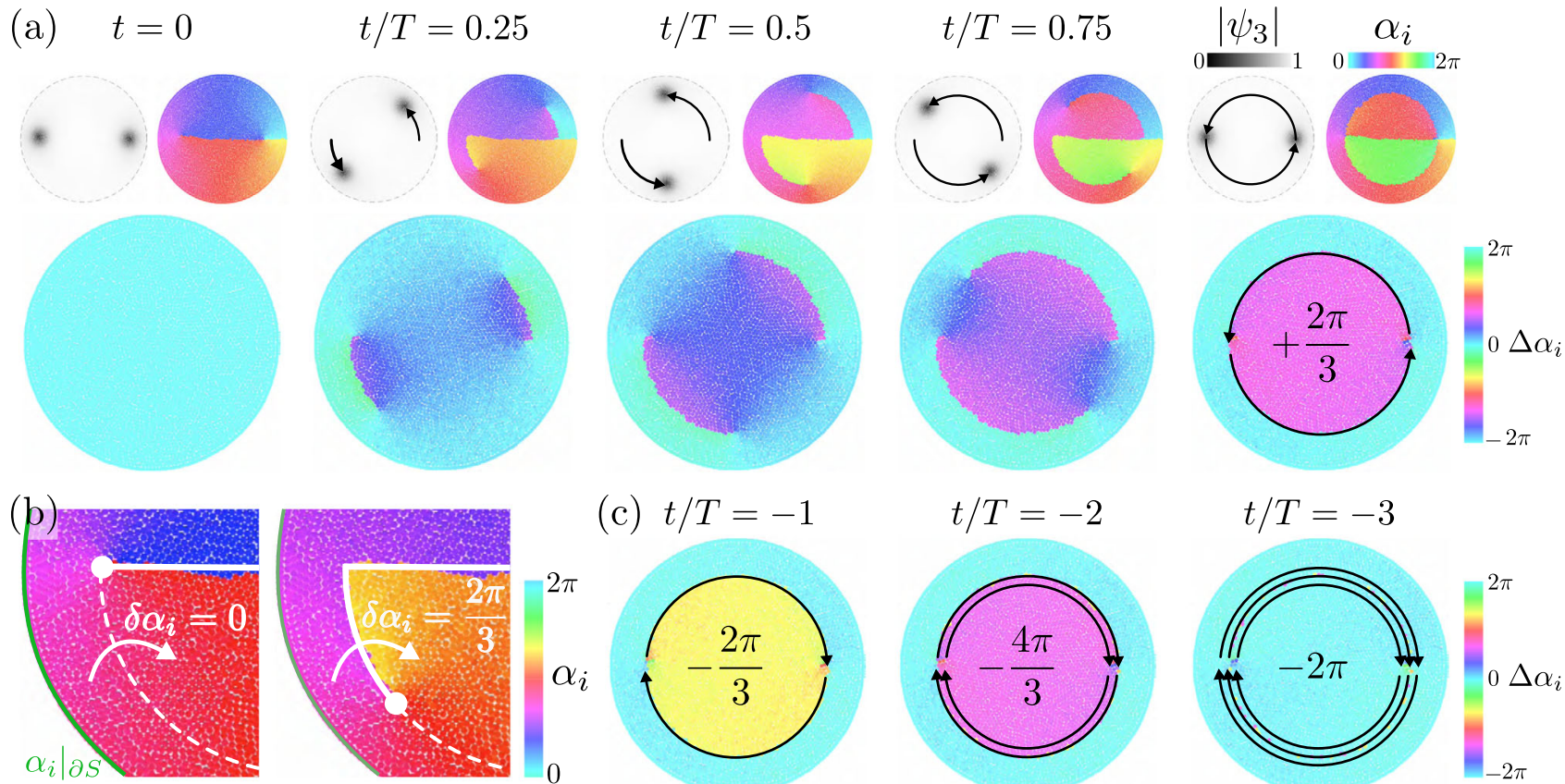

(c) $t / T=-1$

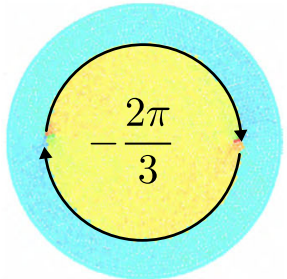

$$
t / T=-2
$$

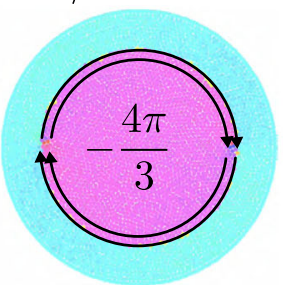

$$
t / T=-3
$$

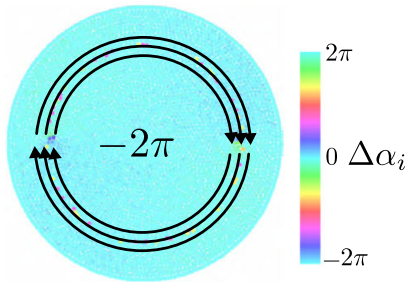

FIG. 3. Defect braiding in $k$-atic particle simulations with aligning interactions $(g>0)$, which gives rise to emergent anyonic exchange symmetries. (a) Pair of symmetric $1 / 3$ defects in the 3 -atic particle model on a unit disk [the final state of Fig. 2(d)] braided counterclockwise through a modulation of the boundary-anchoring profile [Eqs. (17)-(19) with $\theta_{0}=0$ and $t / T \in[0,1]$. Here, $\psi_{3}$ denotes the local 3-atic order parameter [see Fig. 2(b) and Eq. (A9)], and $\Delta \alpha_{i}:=\alpha_{i}(t)-\alpha_{i}(t=0)$ measures the change of a particle's orientation angle. After one defect exchange $(t / T=1$, rightmost images), the domain enclosed by the defect trajectories (black arrows) picks up a globally uniform orientation angle difference $\Delta \alpha_{i}=2 \pi / 3$. (b) Enlarged portion of particle angles $\alpha_{i}$ shown in panel (a) (left, $t / T=0$; right, $t / T=0.25)$ revealing the mechanism by which the inner domain picks up a well-defined angle difference: The orientation of particles near the boundary is held fixed through the boundary anchoring $\left.\alpha_{i}\right|_{\partial S}$. Initially (left), particle angles change continuously $\left(\delta \alpha_{i}=0\right)$ along the radial direction. The $1 / 3$ defects (white dot) are connected by a branch cut (white solid line) across which particle orientation angles $\alpha_{i}$ jump by $2 \pi / 3$. Defect braiding represents a continuous deformation of the branch cut (right), which introduces a discontinuous jump $\delta \alpha_{i}=2 \pi / 3$ relative to (fixed) particle orientations near the disk boundary. (c) Running the braiding protocol backwards leads to clockwise braiding and opposite signs of $\Delta \alpha_{i}$. For the case $k=3$ illustrated here, the system returns to its initial state after three successive braiding steps, in analogy with anyonic excitations in quantum systems (Supplemental Movie 3 [89]). All simulations use the boundary anchoring given in Eqs. (18) and (19) with $a=0.1$ and $D_{r} T=20$; all other parameters are as in Fig. 2(d). 
$[39,47]$ by adiabatically changing the anchoring equations (14) and (17) with anchoring profile $\gamma_{k}$ given in Eq. (18) in both the mean-field description and the particle model. Specifically, we interpret defects carrying the same topological charge per Eq. (5) as "identical particles" and, accordingly, aim to braid an identical defect pair such that the phase of the final state $\Psi_{k}(t=T)$ differs from that of the initial state $\Psi_{k}(t=0)$ by a constant global shift. To this end, it is instructive to first discuss how defect braiding in the microscopic model generates an emergent anyonic exchange symmetry on a subdomain of the disk. In a second step, we show how this can be extended to a protocol where defect exchanges are accompanied by global $k$-atic phase changes.

\section{Braiding-induced anyonic exchange symmetries in the particle model}

We consider a pair of $1 / k$ defects that are initially aligned with the $x$ axis [Fig. 3(a), $t=0$ ]. As discussed above, this configuration can be achieved by imposing the anchoring condition (14) with $m=2$ and $\theta_{\mathrm{a}}=0$ in Eq. (18). To parametrize the exchange of defect positions, we consider the anchoring profile $\gamma_{k}$ given in Eq. (18) with $\theta_{0}=0$ and

$$
\theta_{\mathrm{a}}(t)=\frac{\pi t}{T}
$$

For increasing $t \in[0, T]$ with $T>0$, this leads to a counterclockwise rotation of the preferred localization axis of the defect pair during which the two defects move along complementary semicircles [see $\left|\psi_{3}\right|$ in Fig. 3(a)]. These trajectories eventually enclose a subdomain where particle orientation differences fluctuate around $\Delta \alpha_{i}=\alpha_{i}(T)-$ $\alpha_{i}(0)=2 \pi / k$ [shown in Fig. 3(a) for $k=3$ ], corresponding to an anyonic exchange symmetry [38-40,46,47] of the polar order-parameter field $\psi_{1}$ [Eq. (A9)]. The emergence of this subdomain can be understood as follows: Short-range alignment interactions together with the boundary anchoring $\left.\alpha_{i}\right|_{\partial S}$ essentially fix orientations of particles near the boundary. Consequently, a passing-by branch cut led by a $1 / k$ defect causes a change of the interior local particle orientations by $2 \pi / k$, as shown for $k=3$ in Fig. 3(b).

Braiding clockwise instead, $t \in[0,-T]$, changes local particle angles by $-2 \pi / k$ when defects pass by and consequently leads to a sign flip of $\Delta \alpha_{i}$ [Fig. 3(c)], analogous to the properties of an anyonic exchange symmetry [47]. Finally, by performing $k$ such braids, we demonstrate that the orientation angle change $\Delta \alpha_{i}$ after each braid is quantized in steps of $\pm 2 \pi / k$, despite the
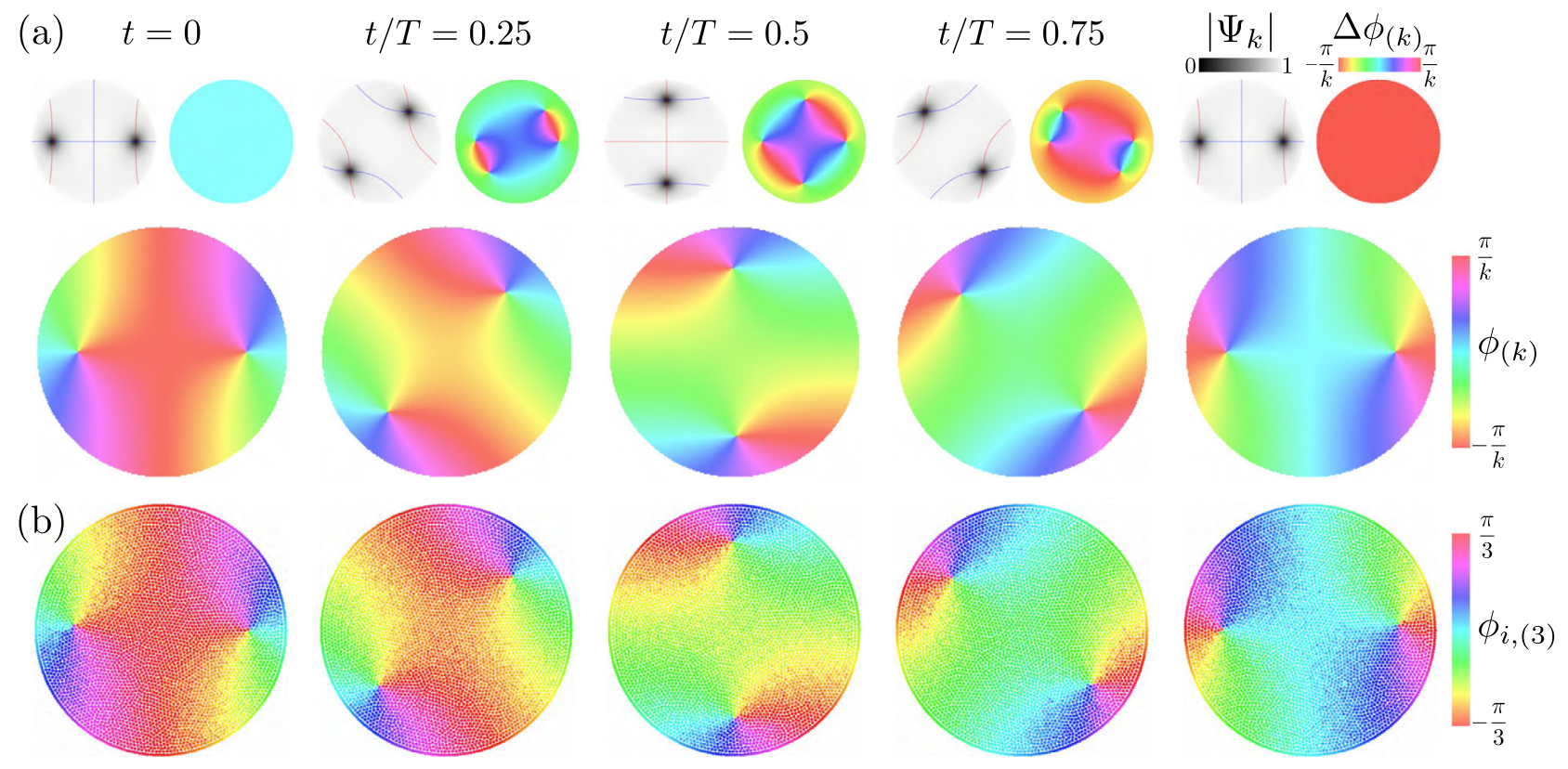

FIG. 4. Demonstration of the braiding protocol in GL mean-field theory and particle simulations with aligning interactions ( $g>0)$ on the unit disk. (a) Pair of identical $1 / k$ defects braided in GL simulations over the interval $t / T=[0,1]$ through a modulation of the boundary anchoring. Red and blue lines indicate $\operatorname{Re} \Psi_{k}=0$ and $\operatorname{Im} \Psi_{k}=0$, respectively. The $k$-atic phase shift $\Delta \phi_{(k)}$ given in Eq. (21) assumes the constant global value $\Delta \phi_{(k)}=\pi / k$ for $t=T$ (top right; Supplemental Movie 4 [89]). The $k$-atic phases shown are exact stationary solutions of the GL, corresponding to perfectly adiabatic braiding with $T \rightarrow \infty$. (b) Particle simulations $\left(D_{r} T=20\right)$ for a braided pair of 1/3 defects, replicating the GL prediction (Supplemental Movie 5 [89]). Simulations use the boundary-anchoring equations (14) (mean field) and (17) (particle model) with the anchoring profile $\gamma_{k}$ defined through Eqs. (18)-(20) with $a=0.1$; all other parameters are as in Fig. 2(d). 
stochasticity in the particle model [Fig. 3(c)]. Indeed, three consecutive braids of 3-atic particles return all particles in the subdomain enclosed by the defect trajectories to their initial orientations (Supplemental Movie 3 [89]).

\section{Defect braiding and global $k$-atic phase changes in mean-field theory and particle model}

Our next goal is to identify a boundary-anchoring protocol for which defect braiding is accompanied by a global $k$-atic phase shift. To this end, particle orientations in the outer annulus, where, so far, $\Delta \alpha_{i}=0$ [Figs. 3(a) and 3(c)], also have to change with the braiding. Adding a constant global angle $\mp 2 \pi / k$ to the boundary-anchoring profile is not sufficient, as it equally affects the whole disk such that the angle difference $\Delta \alpha_{i}= \pm 2 \pi / k$ between the annulus and the domain enclosed by the defect trajectory remains. However, a constant global phase shift of $k$-atic director orientations $\phi_{i,(k)}$ [Eq. (16)] and of the $k$-atic meanfield phase $\phi_{(k)}$ [Eq. (4)] can be achieved by exploiting the fact that for both quantities $-\pi / k$ is identified with $\pi / k$. Specifically, we use the anchoring profile Eq. (18) with $\theta_{\mathrm{a}}(t)$ given in Eq. (19) and

$$
\theta_{0}(t)=\frac{\pi t}{2 T}
$$

As before, this protocol induces a counterclockwise braid that exchanges the two identical defects. However, now Eq. (20) simultaneously modulates the $k$-atic phase $\phi_{(k)}$ within the annulus and the subdomain enclosed by a defect trajectory by $-\pi / k$ such that a globally constant $k$-atic phase difference

$$
\Delta \phi_{(k)}=\frac{1}{k} \arg \left[\Psi_{k}(T) \Psi_{k}^{*}(0)\right]=\frac{\pi}{k}
$$

emerges, as illustrated by stationary solutions of the GL equation for different $t / T$ in Fig. 4(a) (Supplemental Movie 4 [89]). The stochastic particle model undergoes a noisy realization of this texture sequence [Fig. 4(b); Supplemental Movie 5 [89] ] if the same boundary anchoring $\gamma_{k}$ is used in Eq. (17).

More generally, this demonstrates how one can implement an exchange of two identical defects with an accompanying global $k$-atic phase change in the complex order-parameter field of DLCs. We emphasize, however, that a global phase change in the $k$-atic director field necessitates a corresponding phase change at the boundary.

\section{SPONTANEOUS CHIRAL SYMMETRY BREAKING AND BRAIDING IN ANTIALIGNING DLCs $(g<0)$}

Having focused on aligning interactions in the previous section, we now consider $k$-atic DLCs with short-range antialigning interactions $[g<0$; Figs. 1 (a) and $1(\mathrm{~b})]$. In this case, the mean-field model in Eq. (1) takes the form of a Swift-Hohenberg $(\mathrm{SH})$ equation for the complex order parameter $\Psi_{k}$. For boundary anchorings that impose a topological charge of $1 / k$ on a unit disk, the $\mathrm{SH}$ equation predicts a spontaneous chiral symmetry breaking of texture patterns that is also observed in the microscopic particle model. We then show that this chiral symmetry breaking can be understood analytically by constructing a stationary solution to the linearized complex SH equation. Last but not least, to demonstrate the versatility of the braiding protocol from Sec. IV C, we perform a braiding operation for a defect pair in antialigning DLCs in both the SH equation and the microscopic model.

\section{A. SH mean-field theory for antialigning $\boldsymbol{k}$-atics}

We consider the microscopic model Eq. (6) with $g<0$, which favors antialigning configurations of nearby $k$-atic particle directors. For a small but finite interaction range $R_{\alpha}>0$, the mean-field dynamics of the $k$-atic mode can be approximated by (Appendix A)

$$
\bar{\tau} \partial_{t} \psi_{k} \approx h_{k}-\left(\beta_{1} R_{\alpha}^{2} \nabla^{2}+\beta_{2} R_{\alpha}^{4} \nabla^{2} \nabla^{2}\right) \psi_{k},
$$

where the homogeneous terms $h_{k}$ were defined previously in Eq. (10). The coefficients $\beta_{1}$ and $\beta_{2}$ in Eq. (22) depend on the spatial interaction kernel of the microscopic model. Assuming, as before, equally weighted interactions between particles within a neighborhood of radius $R_{\alpha}$, the kernel determines the dispersion relation for perturbations of the $k$-atic mode $\psi_{k} \sim f_{k}$ around the disordered state $f_{k}=0$, which is approximated by the parameters $\beta_{1}$ and $\beta_{2}$ (Appendix A 2).

The coarse-graining result, Eq. (22), implies a meanfield model for antialigning DLCs of the general form, Eq. (1), with

$$
\mathcal{L}\left(\nabla^{2}\right)=-L_{1}^{2} \nabla^{2}-L_{2}^{4} \nabla^{2} \nabla^{2},
$$

corresponding to a SH equation [55] for the complex order parameter $\Psi_{k}$. Equation (22) specifies the mean-field parameters $L_{1}$ and $L_{2}$ in Eq. (23) in terms of the interaction radius $R_{\alpha}$ as

$$
L_{1} \simeq \beta_{1}^{1 / 2} R_{\alpha}, \quad L_{2} \simeq \beta_{2}^{1 / 4} R_{\alpha} .
$$

The relaxation dynamics corresponding to such a meanfield theory is generated by a Landau-Brazovskii energy $\mathcal{E}_{k}$ for the complex order parameter $\Psi_{k}$ [48] (Appendix C 1). Furthermore, the ratio $L_{2}^{2} / L_{1}$ signals an emergent mesoscopic length scale in the phase field, arising from the competition between antialigning particle interactions. In particular, we expect, for the dynamic equation (1) with $\mathcal{L}\left(\nabla^{2}\right)$ given in Eq. (23), finite wavelength instabilities at wave-vector amplitude $q_{0}^{2}$, when $A<A_{*}$, where 


$$
q_{0}^{2}=\frac{L_{1}^{2}}{2 L_{2}^{4}}, \quad A_{*}=\frac{L_{1}^{4}}{4 L_{2}^{4}},
$$

a prediction that is validated below in both the particle model and in the quantitatively mapped complex $\mathrm{SH}$ equation.

\section{B. Spontaneous chiral symmetry breaking of texture patterns}

Even when microscopic particles are achiral, the interplay between antialigning particle interactions and boundary conditions can give rise to an interesting spontaneous chiral symmetry-breaking phenomenon. In the following, this is first demonstrated using the nonlinear dynamics of threefold symmetric particles described by $k=3$ and $g<0$ in Eq. (6) and comparing them with predictions from the corresponding SH mean-field equation (1) with operator equation (23). Finally, we describe analytic stationary solutions of the linearized complex $\mathrm{SH}$ equation that recapitulate the observed patterns, as well as the bifurcation into the chiral symmetry-breaking transition.

\section{Pattern formation on the unit disk}

We first consider a topologically trivial boundary anchoring corresponding to $m=0$ in Eq. (14). In this case, the SH mean-field theory predicts the existence of defect-free, azimuthally symmetric, stationary states that can indeed be observed in simulations of the antialigning particle model [Fig. 5(a), $m=0$ ]. However, although such defect-free states are long-lived in the presence of noise, they only form from suitably prepatterned initial conditions (a)
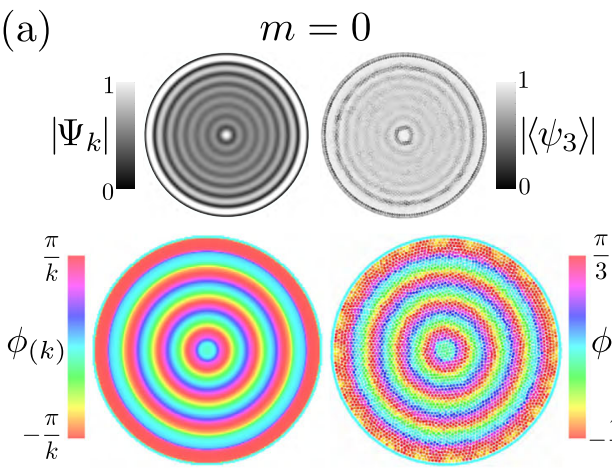

(b)

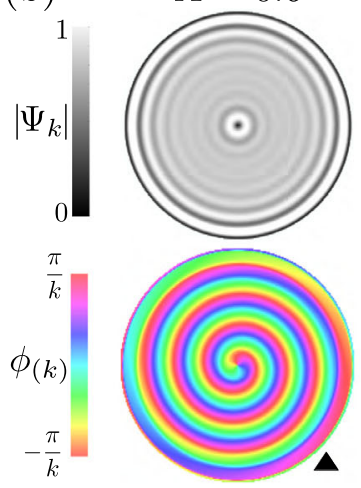

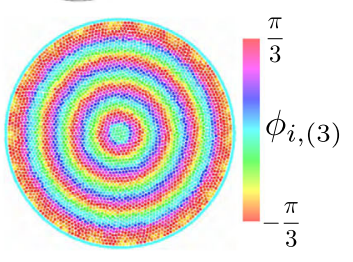

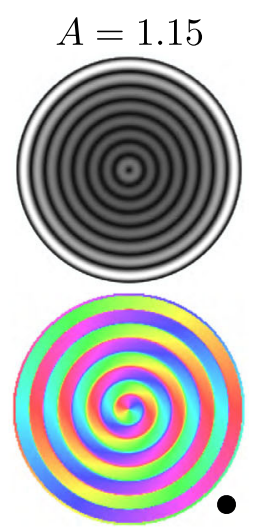

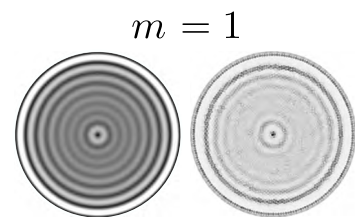
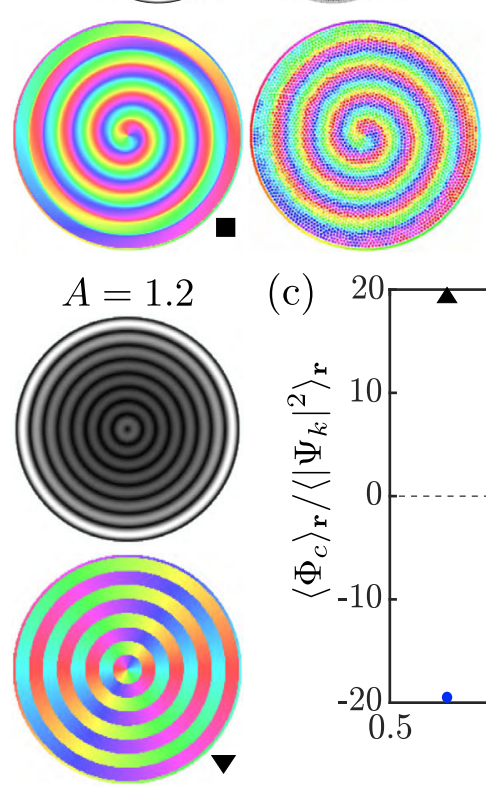

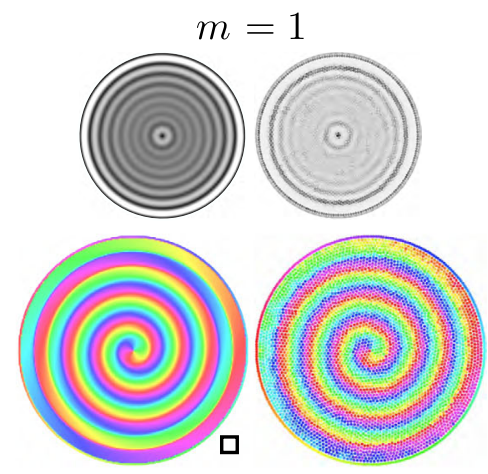

(c)

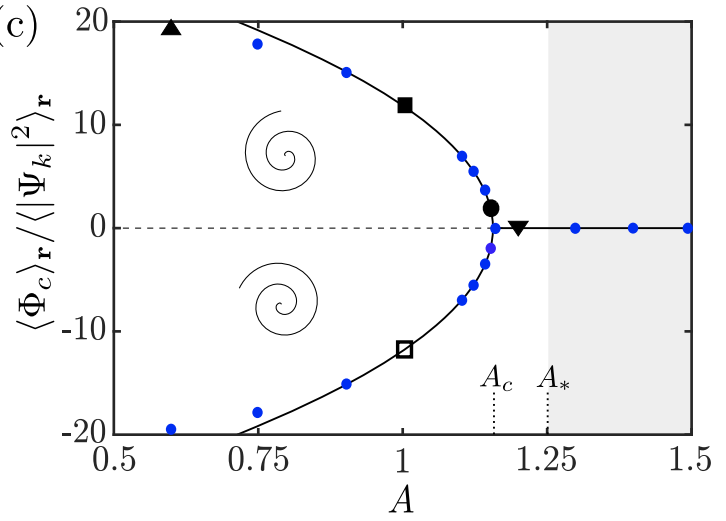

FIG. 5. Chiral symmetry breaking in phase field textures of the SH mean-field theory and particle simulations with antialigning interactions $(g<0)$ on a unit disk. (a) Stationary order parameters (top) and phase-field solutions (bottom) for the SH mean-field equation (1) with $\mathcal{L}=-L_{1}^{2} \nabla^{2}-L_{2}^{4}\left(\nabla^{2}\right)^{2}$ and the particle model equation (6) with antialigning interactions using the boundaryanchoring equations (14) and (17), respectively, with $\gamma_{k}=\theta$. A stationary defect-free axisymmetric state exists for topologically trivial boundary anchoring with $m=0$. For $m=1$, chiral textures with $1 / k$ defects at the center form spontaneously in both mean-field and particle simulations (Supplemental Movie 6 [89]). Mean-field simulation parameters are $A=B=1, L_{1}$, and $L_{2}$ as defined in Eq. (24), with $\beta_{1}=0.1, \beta_{2}=0.002$ (characteristic wave number $q_{0}=25$ ), and $R_{\alpha}=0.2$. Particle simulation parameters are $k=3, g=-1$, and all other parameters are as in Fig. 2(d). (b) Examples of the spontaneously formed stationary solution of the complex SH equation for $m=1$ and selected values of the control parameter $A$ with parameters identical to those in panel (a). (c) Phase-chirality parameter $\Phi_{c}$ defined in Eq. (26) characterizing the spontaneous symmetry breaking into chiral textures of different handedness $\left(\langle\cdot\rangle_{\mathbf{r}}\right.$ denotes spatial averages). The bulk dynamics of the complex SH equation is linearly stable for $A>A_{*}$ (gray-shaded region), but achiral ring patterns remain because of the boundary-anchoring equation (14) with $\left|\Psi_{k}\right|_{\partial S}=1$. Phase-chirality parameters measured in additional simulations for different values of $A$ (small blue dots) approach the curve $\sim \pm \sqrt{A_{c}-A}$ for $A \searrow A_{c}$, with $A_{c} \approx 1.155$, consistent with a supercritical pitchfork bifurcation. 
(Appendix D), whereas random initial conditions typically lead to ring-shaped patterns that are intersected by chains of $\pm 1 / k$-point defects with zero topological net charge [Fig. 10(c)].

For a boundary-anchoring equation (14) with $\gamma_{k}=\theta$ and $m=1$, the SH mean-field theory predicts the spontaneous formation of a spiral-shaped chiral texture with a $1 / k$ defect at the center of the disk, while the $k$-atic order parameter $\left|\Psi_{k}\right|$ maintains an azimuthal symmetry. Again, the particle model confirms this prediction [Fig. 5(a), right, and Supplemental Movie 5 [89]]. Such chiral textures are reminiscent of spiral patterns that have recently been observed theoretically and experimentally in cholesteric LCs $[74,75]$. However, the latter arise from chiral microscopic interactions, while spiral texture patterns in our system arise from a spontaneous symmetry breaking among isotropically interacting achiral particles.

\section{Characterization of the chiral symmetry-breaking transition}

Upon varying $A$ in simulations of the complex $\mathrm{SH}$ equation, while keeping all other parameters fixed, we find that textures become chiral only below a critical value $A_{c}$, but they remain azimuthally symmetric if $A>A_{c}$ [Figs. 5(b) and 5(c)]. To quantify this transition, we introduce the phase-chirality parameter (Appendix C 2 b)

$$
\Phi_{c}=k\left|\Psi_{k}\right|^{2} \mathbf{e}_{r} \cdot \nabla \phi_{(k)} .
$$

This quantity characterizes chiral signatures in textures around a defect by measuring the radial contributions in the $k$-atic phase gradient. In particular, in our system, $\Phi_{c}>0$ $\left(\Phi_{c}<0\right)$ indicates the presence of right-handed (lefthanded) texture spirals leading into the defect [Fig. 5(c)]. Averaging $\Phi_{c}$ across the disc domain, we observe a continuous transition from achiral to chiral textures at $A_{c}$. The transition exhibits the characteristics of a supercritical pitchfork bifurcation with left- and right-handed texture patterns forming with equal probability from random initial conditions [Fig. 5(c)]. Note that the transition point $A_{c}$ tends, on an infinite domain, to the linear stability threshold $A_{*}$ (see Sec. V B 4).

\section{Wavelength doubling in amplitude and phase patterns}

For all stationary solutions shown in Fig. 5(a), the wavelength of texture patterns is in quantitative agreement with the mean-field prediction $\lambda_{0}=2 \pi / q_{0} \approx 0.25$. This can be seen from the $k$-atic phases $\phi_{(k)}$ and $\phi_{i,(3)}$ that cycle about $1 / \lambda_{0} \approx 4$ times through $2 \pi / k$ between the boundary and center of the unit disk. Interestingly, the wavelength of texture patterns is twice as large as the wavelength of orderparameter amplitude patterns in both the particle model $\left(\left|\left\langle\psi_{3}\right\rangle\right|\right)$ and the mean-field theory $\left(\left|\Psi_{k}\right|\right)$. In the particle model, this can be rationalized as follows: Two, single, antialigning particles interacting via Eq. (6) $(g<0)$ are stationary for a director angle difference of $\pi / k$. However, when antialigning interactions are present over a finite range and include several particles, it becomes energetically favorable to form finite-sized regions, each with an approximately constant director orientation (corresponding to high orientational order $\left.\left|\left\langle\psi_{3}\right\rangle\right|\right)$ but with a difference of $\pi / k$ to director orientations in the directly neighboring region. As a result, one cycle of the $k$-atic phase through $2 \pi / k$ contains two regions of high order, and consequently, the wavelength of amplitude patterns is half the wavelength of texture patterns. On the unit disk, regions of high order form annuli due to the boundary anchoring [Figs. 5 and 10(c)], whereas on periodic domains, they are given by checkerboard patterns (Fig. 11). The meanfield theory recapitulates these nontrivial consequences of microscopic antialignment in all cases with good quantitative agreement, which can be understood from analytic stationary solutions of the linearized complex SH equation.

\section{Analytic solutions of the complex SH equation}

All observations from Sec. V B can be recapitulated by analytic stationary solutions near the linear instability. Specifically, we show in Appendix C 2 that exact solutions of the linearized complex SH equation

$$
A \Psi_{k}+L_{1}^{2} \nabla^{2} \Psi_{k}+L_{2}^{4} \nabla^{2} \nabla^{2} \Psi_{k}=0
$$

can be found by solving the equivalent bi-Helmholtz equation

$$
\left(\nabla^{2}+q_{+}^{2}\right)\left(\nabla^{2}+q_{-}^{2}\right) \Psi_{k}=0,
$$

where $q_{ \pm}^{2}=q_{0}^{2} \pm \sqrt{\Delta A} / L_{2}^{2}$, with $\Delta A=A_{*}-A$ (Appendix C 2), and $q_{0}$ and $A_{*}$ are given in Eq. (25). Solutions of Eq. (27) in polar coordinates $(r, \theta)$ take the form

$$
\Psi_{k}(r, \theta)=\sum_{m=0}^{\infty}\left[\mu_{m} J_{m}\left(q_{-} r\right)+\nu_{m} J_{m}\left(q_{+} r\right)\right] e^{i m \theta},
$$

where $J_{m}(x)$ are Bessel functions of the first kind, and $\mu_{m}$ and $\nu_{m}$ denote complex integration constants. Each mode $m$ in Eq. (28) represents an azimuthally symmetric amplitude pattern $\left|\Psi_{k}\right|$ that harbors a defect of topological charge $q_{\mathrm{d}}=$ $m / k$ at $r=0$, consistent with stationary states shown in Figs. 5(a) and 5(b).

At the critical point $A=A_{*}$, where $q_{ \pm}=q_{0}$, all modes in Eq. (28) are of the form $\Psi_{k} \sim J_{m}\left(q_{0} r\right) e^{i m \theta}$. Such fields represent concentric annuli of phase patterns with wavelength of about $2 \pi / q_{0}$ and amplitude patterns with half this wavelength, about $\pi / q_{0}$ [Figs. 6(a) and 6(b), Appendix C 2 a]. Beyond the critical point $A<A_{*}$, where $q_{+} \neq q_{-}$, the mode $m=1$ in Eq. (28) can represent rightand left-handed chiral texture patterns $\phi_{(k)}$ [Fig. 6(b), 

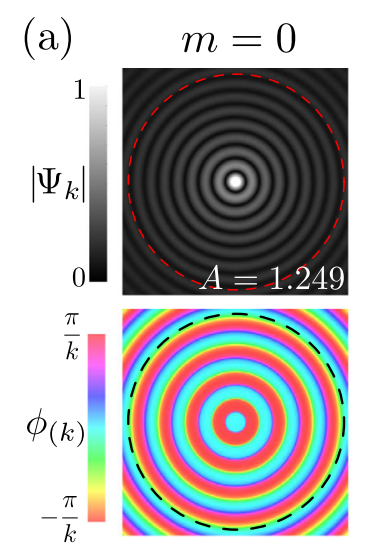

(b)
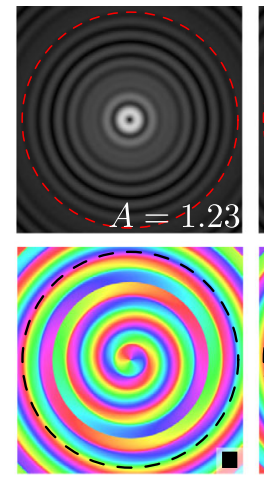

$m=1$
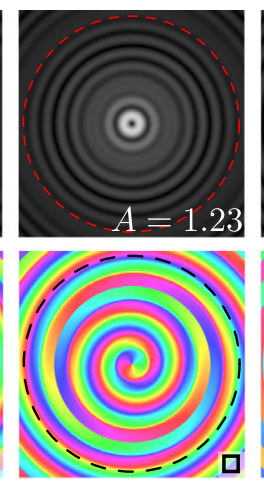
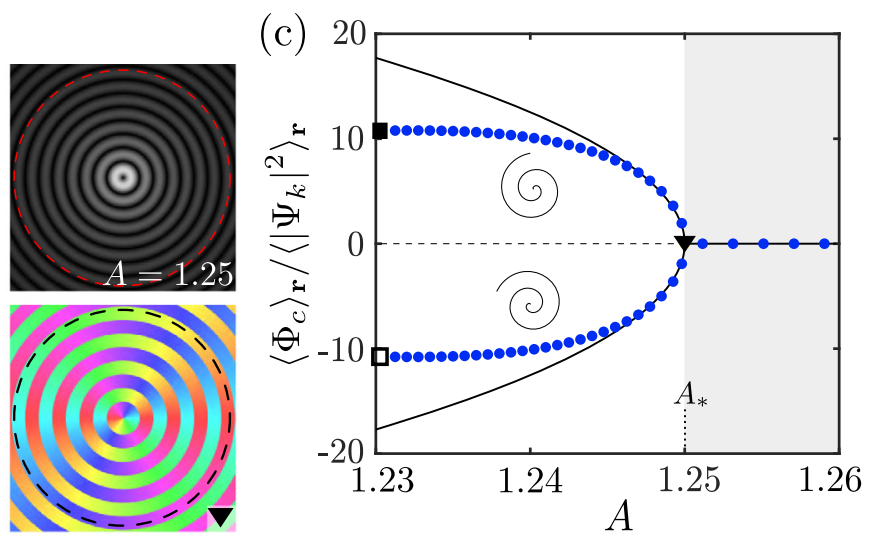

FIG. 6. Analytic solutions of the linearized complex SH equation on infinite domains explain pattern formation and chiral symmetry breaking. (a) Defect-free solution [Eq. (28) with $m=0$ and $\mu_{0}=\nu_{0}^{*}=e^{i \pi / 4}$ ] recapitulates the wavelength doubling between amplitude and phase patterns [Fig. 5(a), Appendix C 2 a]. (b) Examples from the family of analytic solutions, Eq. (28), with $m=1, \mu_{1}=e^{i \pi / 4}$, and $\nu_{1}= \pm e^{-i \pi / 4}$ (" + " is for right-handed spirals; "-" is for left-handed spirals), showing bifurcation into chiral texture patterns, as quantified in panel (c), when the control parameter $A$ becomes smaller than the critical value $A_{*}$ [see Eq. (25)]. All fields in panels (a) and (b) use the same length scales $L_{1}$ and $L_{2}$ as in Fig. 5 and are compatible with the director anchoring equation (14) for $\gamma_{k}=\theta$ at a unit disk boundary (red and black dashed lines). (c) Phase chirality with $\Phi_{c}$ defined in Eq. (26), evaluated on a unit disk centered at $r=0$ for analytic solutions (blue dots). Black symbols indicate exemplary solutions shown in (b). Solid black lines depicts a fit $\propto \sqrt{A_{*}-A}$ near the critical value $A_{*}$. Compared to these analytic solutions, numerical solutions of the fully nonlinear SH equation on a finite domain exhibit a small shift in the critical value $A_{c}$ of the bifurcation [Fig. 5(c), $A_{c}<A_{*}$ ], reflecting additional effects from the nonlinearity $B\left|\Psi_{k}\right|^{2} \Psi_{k}$ and from boundary conditions.

(a) $t=0$
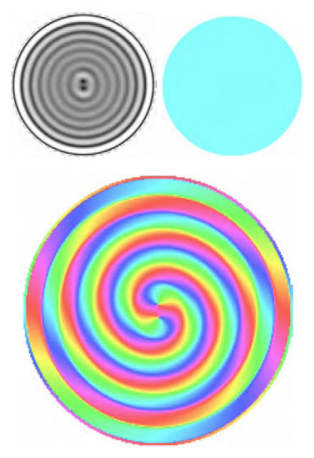

(b)

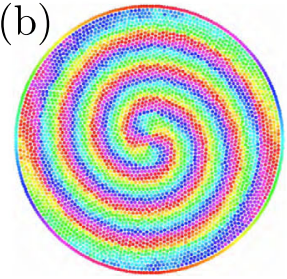

$t / T=0.25$
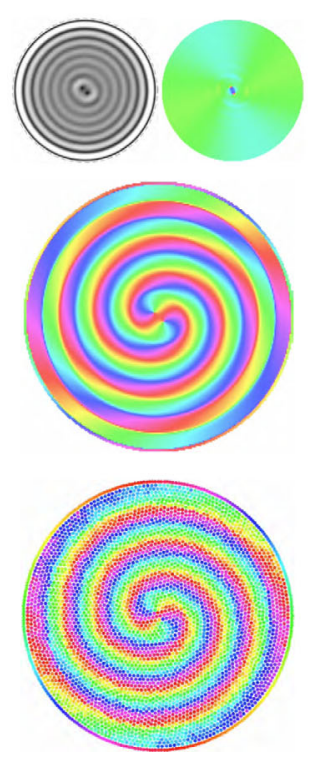

$t / T=0.5$
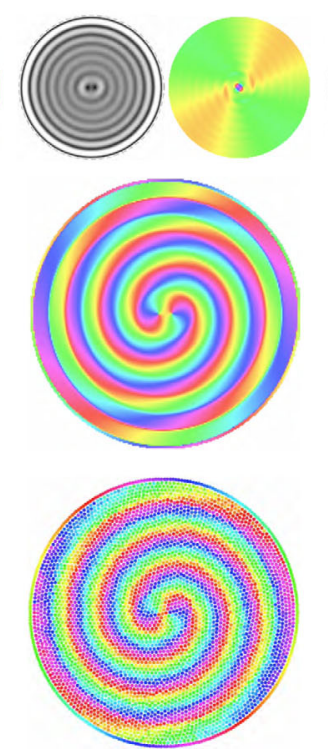
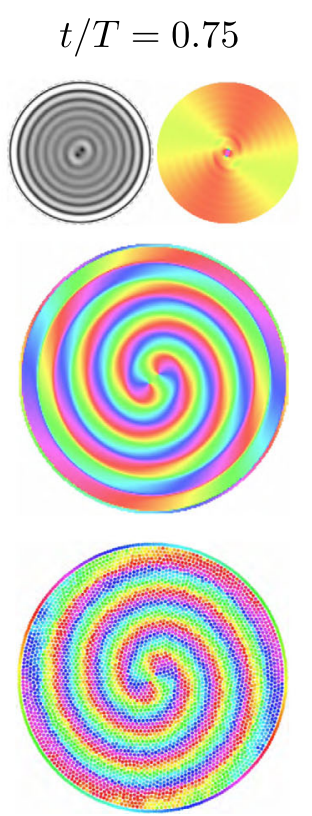
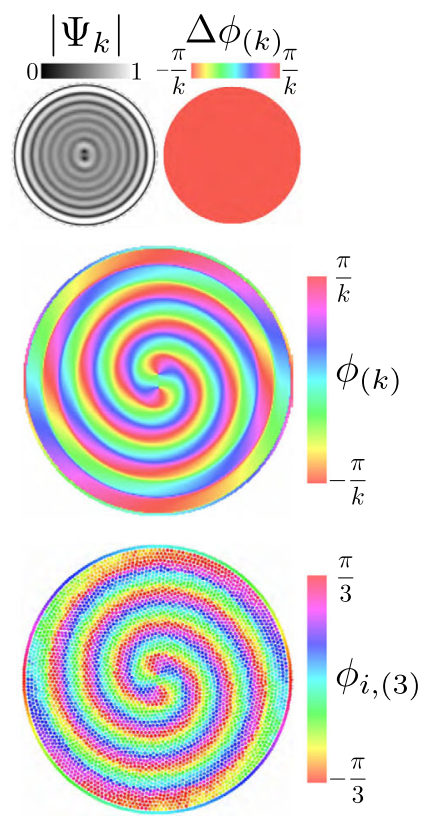

FIG. 7. Demonstration of the braiding protocol in (a) the SH mean-field theory and (b) particle simulations with antialignment interactions $(g<0)$ on the unit disk (Supplemental Movie 7 and 8 [89]). Boundary-anchoring equations (14) (mean-field) and (17) (particle model) with $m=2$ impose a total defect charge of $2 / k$ on the domain, supporting two stable $1 / k$ point defects near the disc center and outwards spiraling textures. Similar to Fig. 4, we applied the boundary-controlled braiding protocol with $\gamma_{k}$ defined through Eqs. (18)-(20) and $a=0.2$, while using the model parameters from Fig. 5. The $k$-atic mean-field phase fields in (a) are exact stationary solutions of the complex SH equation, corresponding to perfectly adiabatic braiding with $T \rightarrow \infty$. In both mean-field and particle simulations, the initial defect-pair state does not form spontaneously from random initial conditions but can be robustly generated as a stationary state from initial conditions that are sufficiently close to a double-spiral texture (Appendix D). The braiding protocol starts at $t=0$ by modulating the boundary anchoring as described in Sec. IV C. At the end of the braiding process, the two point defects have exchanged their position, and the director field has acquired a constant global $k$-atic phase shift $\Delta \phi_{(k)}=\pi / k$ in the SH equation (a) and of $\pi / 3$ in the antialigning particle model with $k=3$ (b). 
Appendix C 2 b], while amplitude patterns $\left|\Psi_{k}\right|$ maintain azimuthal symmetry. These key features in both achiral and chiral stationary analytic solutions recapitulate our findings from numerical solutions of the fully nonlinear mean-field theory (Fig. 5), including the pitchfork bifurcation of stationary solutions beyond a critical control parameter threshold $A_{*}$ into chiral texture patterns [Fig. 6(c)].

\section{Braiding of chiral texture patterns}

Similar to their achiral counterparts, the $k$-atic fields describing chiral defect pairs in antialigning DLCs can also be braided by applying the adiabatic protocol from Sec. IV C. To demonstrate this, in both SH mean-field and particle simulations, we use the boundary condition Eq. (14) with $m=2$ and fix the anchoring profile $\gamma_{k}$ from Eq. (18) with $a=0.2$. This boundary condition now stabilizes a vertically oriented pair of $1 / k$ defects near the disk center, which is surrounded by texture spirals that intertwine towards the boundary (Fig. 7, $t=0$ ). To prepare this initial state in the continuum and particle simulations, one can let the system relax from suitably prepatterned textures (Appendix D). The resulting two-defect state is stable and can be robustly braided using the anchoring profile parametrization given in Eq. (20). Snapshots of the braiding sequences, selected from Supplemental Movies 7 and 8 [89], are shown in Fig. 7. At the end of the braiding operation, the defects have exchanged their positions, and the complex order-parameter field $\Psi_{k}$ has acquired a constant global phase shift $\Delta \phi_{(k)}=\pi / k$ [Eq. (21)].

\section{CONCLUSIONS}

The above analysis shows that the relaxation behavior and adiabatic manipulation of 2D liquid crystals composed of $k$-fold symmetric particles can be accurately described within a unified mean-field theory for a complex orderparameter field. Because of the generic character of the underlying particle model, which merely assumed overdamped short-range $X Y$-type interactions on a disordered lattice, we expect that the above ideas can be experimentally implemented and tested in different ways. Promising candidates include colloidal systems [3,4] with predefined symmetries and controllable steric $[8,10,12,14,15]$, magnetic $[52,76]$, or chemical $[9,77]$ interactions. The main experimental challenge will be to enforce the required short-range orientational interactions while simultaneously suppressing positional order. This could, for example, be achieved using weakly multidisperse $k$-atic platelet systems, similar to those realized in Refs. [8,14]. Other promising candidate systems could be thin films of threefold symmetric molecules [22] or $k$-fold symmetric DNA-origami structures [49,50], building on recently developed experimental techniques [51] for the assembly and control of DNA-origami-based liquid crystals.

From a general theoretical perspective, $k$-atic DLC systems [34] provide a useful classical framework for studying and visualizing fractional topological excitations and their exchange properties. Since the energetic correspondence with quantum fluids only holds at the mean-field level, it remains an interesting open question whether and how the statistical properties of fractional defects in DLCs depend on their braiding behavior and if, for example, this may affect their rheological properties. In addition, the above results suggest multiple directions for future research, including generalizations to passive and active $k$-atic hydrodynamic systems [28,78] in two and three dimensions, which can be expected to exhibit new forms of energy transport and turbulence $[18,79]$.

\section{ACKNOWLEDGMENTS}

We thank Vili Heinonen, Martin Zwierlein, and Mehran Kardar for helpful discussions and insightful comments. This work was supported by a Longterm Fellowship from the European Molecular Biology Organization (EMBO ALTF 528-2019, A. M.), a Postdoctoral Research Fellowship from the Deutsche Forschungsgemeinschaft (DFG Project No. 431144836, A. M.), a Complex Systems Scholar Award from the James S. Mc-Donnell Foundation (J. D.), and the Robert E. Collins Distinguished Scholarship Fund (J. D.).

\section{APPENDIX A: COARSE GRAINING OF THE MICROSCOPIC MODEL}

We describe the coarse graining of the minimal microscopic model, Eq. (6), and show how spatial interactions with finite range $R_{\alpha}$ give rise to the operators $\mathcal{L}=R_{\alpha}^{2} \nabla^{2} / 8$ and $\mathcal{L}=-\beta_{1} R_{\alpha}^{2} \nabla^{2}-\beta_{2} R_{\alpha}^{4}\left(\nabla^{2}\right)^{2}$ in the coarse-grained dynamics, Eqs. (12) and (22), respectively.

\section{Hierarchy of mode equations and linearization}

We follow the standard coarse-graining approach [67-69] — by using Itô calculus, neglecting multiplicative noise terms, and factorizing pair correlations - to derive a dynamic equation for the one-particle probability density function $f(\alpha, \mathbf{r}, t)$ from the microscopic model, Eq. (6). For a general interaction kernel $\hat{I}(\mathbf{r})$ that describes how the orientational interactions with neighboring particles are spatially weighted, this equation takes the form

$$
\partial_{t} f(\mathbf{r}, \alpha, t)=\frac{g}{\pi R_{\alpha}^{2}} \partial_{\alpha} \iint d \alpha^{\prime} d \mathbf{r}^{\prime} f(\mathbf{r}, \alpha, t) f\left(\mathbf{r}^{\prime}, \alpha^{\prime}, t\right) \sin \left[k\left(\alpha-\alpha^{\prime}\right)\right] \hat{I}\left(\mathbf{r}-\mathbf{r}^{\prime}\right)+D_{r} \partial_{\alpha}^{2} f .
$$


It is convenient to define an effective, normalized interaction kernel as $I(\mathbf{r})=\hat{I}(\mathbf{r}) /\left(\pi R_{\alpha}^{2}\right)$. Using the Fourier representations

$$
\begin{gathered}
f(\mathbf{r}, \alpha, t)=\frac{1}{(2 \pi)^{3}} \sum_{n \in \mathbb{Z}} \int d \mathbf{q} \tilde{f}_{n}(\mathbf{q}, t) e^{-i(n \alpha+\mathbf{r} \cdot \mathbf{q})}, \\
I(\mathbf{r})=\frac{1}{(2 \pi)^{2}} \int d \mathbf{q} \tilde{I}(\mathbf{q}) e^{-i \mathbf{r} \cdot \mathbf{q}}
\end{gathered}
$$

in Eq. (A1), we can find a hierarchy of coupled dynamic equations for the modes

$$
f_{n}(\mathbf{r}, t)=\frac{1}{(2 \pi)^{2}} \int d \mathbf{q} \tilde{f}_{n}(\mathbf{q}, t) e^{-i \mathbf{r} \cdot \mathbf{q}}
$$

For $k$-atic interactions as given in the microscopic model, Eq. (6), this hierarchy takes the form

$$
\partial_{t} f_{n}(\mathbf{r}, t)=\frac{g n}{8 \pi^{2}} \int d \mathbf{q}\left[f_{n-k}(\mathbf{r}, t) \tilde{f}_{k}(\mathbf{q}, t)-f_{n+k}(\mathbf{r}, t) \tilde{f}_{-k}(\mathbf{q}, t)\right] \tilde{I}(\mathbf{q}) e^{-i \mathbf{r} \cdot \mathbf{q}}-D_{r} n^{2} f_{n}(\mathbf{r}, t),
$$

where, for convenience, we choose a mixed representation in terms of $f_{n}(\mathbf{r}, t)$ and $\tilde{f}_{k}(\mathbf{q}, t)$.

\section{a. Pointwise interactions}

We first discuss the limit of pointwise interactions, corresponding to $I(\mathbf{r})=\delta(\mathbf{r})$ and $\tilde{I}=1$, which has been widely used in models that contain polar $(k=1)$ and nematic $(k=2)$ alignment interactions $[68,80,81]$ as given in Eq. (6). In this case, Eq. (A5) simplify to a spatially homogeneous system of equations,

$$
\partial_{t} f_{n}=\frac{g n}{2}\left(f_{n-k} f_{k}-f_{n+k} f_{-k}\right)-D_{r} n^{2} f_{n} .
$$

This system can be further split into a subset of coupled equations for the modes $n=j k$ with integers $j \neq 0$,

$\partial_{t} f_{j k}=\frac{g j k}{2}\left(f_{(j-1) k} f_{k}-f_{(j+1) k} f_{-k}\right)-D_{r}(j k)^{2} f_{j k}$,

and a system of equations for the modes $f_{n}$ with $n \neq j k$, where the latter modes always vanish at long times. We then generalize the standard closure assumption of a fast relaxation of the next coupled mode [68] to the case of a $k$-atic system, which corresponds to assuming $\partial_{t} f_{2 k}=0$ and $f_{s k}=0$ for integers $s \geq 3$. From the system of equations (A7), we find, in this case, a steady-state value for $f_{2 k}$ and, consequently, the closed coarse-grained dynamics
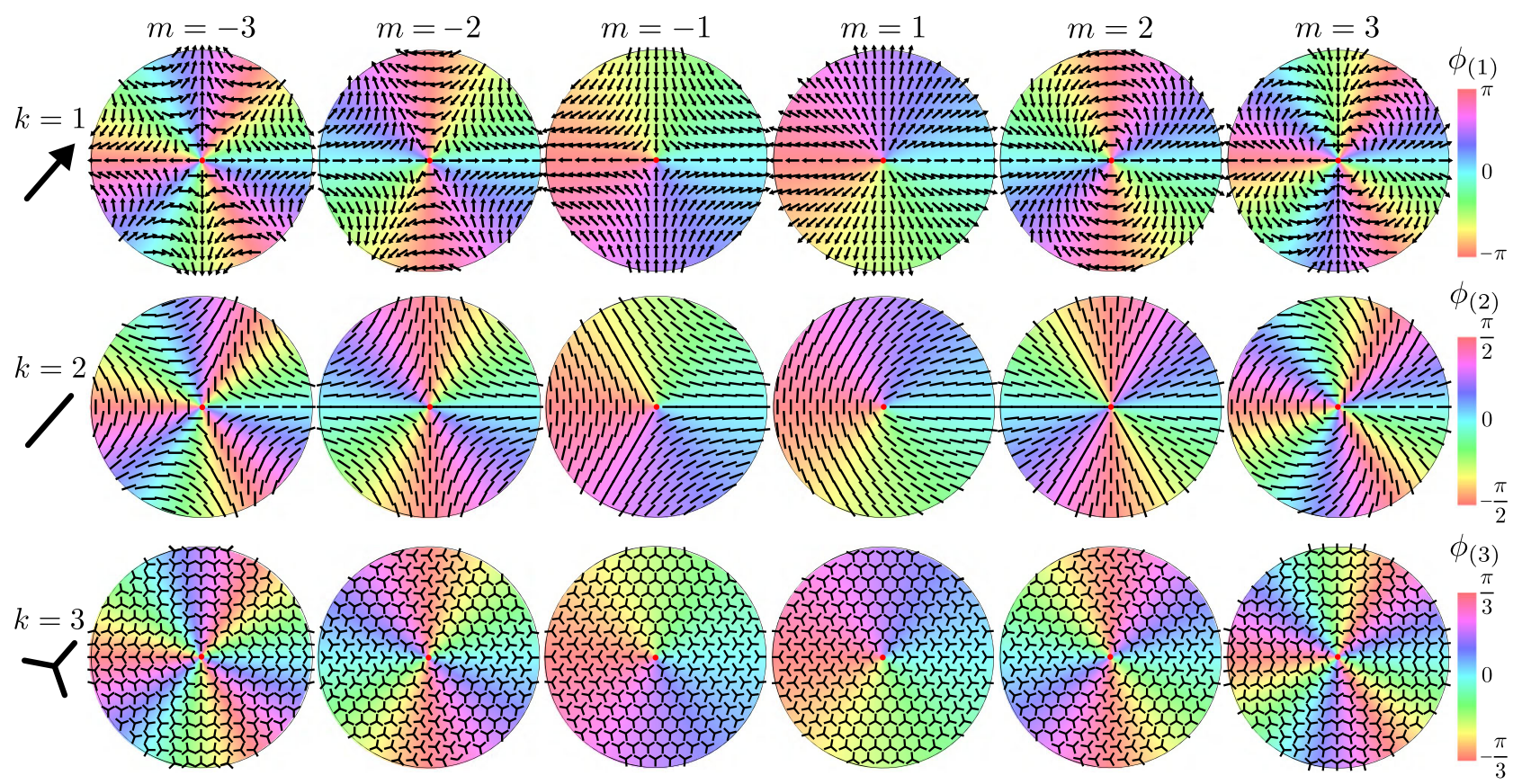

FIG. 8. Visualization of defects formed by polar $(k=1)$, nematic $(k=2)$, and triatic $(k=3)$ particles and their $k$-atic phases $\phi_{(k)}$ for varying winding numbers $m$. The topological charge defined in Eq. (5) of each of the defects is $q_{\mathrm{d}}=m / k$. 


$$
\partial_{t} f_{k}=\frac{g \rho k}{2}\left(1-\frac{2 D_{r} k}{g \rho}\right) f_{k}-\frac{g^{2}}{8 D_{r}}\left|f_{k}\right|^{2} f_{k} .
$$

Using the dimensionless $k$-atic mode $\psi_{k}=f_{k} / \rho$ in Eq. (A8), we arrive at the final coarse-graining result given in Eq. (10).

\section{b. $\boldsymbol{k}$-atic order parameter in particle simulations}

To approximate the normalized $k$-atic mode $\psi_{k}=f_{k} / \rho$ in particle simulations, we use the classical $k$-atic order parameter [2]

$$
\psi_{k}\left(\mathbf{r}_{i}\right)=\frac{1}{\left|\mathcal{N}_{i}\right|} \sum_{j \in \mathcal{N}_{i}} e^{i k \alpha_{j}},
$$

where the sum is evaluated with respect to all particles $j$ within a neighborhood $\mathcal{N}_{i}$ of particle $i$. This can be motivated as follows: Using Eqs. (7) and (8), and ignoring time for brevity, we have

$$
\begin{aligned}
f_{k}\left(\mathbf{r}_{i}\right) & =\int_{0}^{2 \pi} d \alpha \sum_{j}\left\langle\delta\left(\mathbf{r}_{i}-\mathbf{r}_{j}\right) \delta\left(\alpha-\alpha_{j}\right)\right\rangle e^{i k \alpha} \\
& \approx \frac{1}{\pi R_{i}^{2}}\left\langle\int_{0}^{2 \pi} d \alpha \sum_{j \in \mathcal{N}_{i}} \delta\left(\alpha-\alpha_{j}\right) e^{i k \alpha}\right\rangle \\
& \approx \rho\left\langle\psi_{k}\left(\mathbf{r}_{i}\right)\right\rangle .
\end{aligned}
$$

Here, $R_{i}$ denotes the neighborhood radius, and in the last step, we have used the fact that particles are homogeneously distributed, such that $\left|\mathcal{N}_{i}\right| /\left(\pi R_{i}^{2}\right) \approx \rho$. To compute the order parameter $\left\langle\psi_{k}\left(\mathbf{r}_{i}\right)\right\rangle$ with $\psi_{k}\left(\mathbf{r}_{i}\right)$ defined in Eq. (A8) for stationary states [Figs. 2(a), 3(a), 5(a), 10, and 11], we have replaced the Gaussian white-noise average by temporal averages.

\section{c. Linearization with an arbitrary interaction kernel}

For fixed, homogeneous particle number density $\rho=f_{0}$, the mode coupling terms under the integral in the system of equations (A5) only contain a linear contribution when $|n|=k$. As a result, the full linearization of Eq. (A5) around $f_{n}=0$ is, for $|n| \neq k$, simply given by

$$
\partial_{t} f_{n}=-D_{r} n^{2} f_{n},
$$

and for $|n|=k$, it reads

$$
\partial_{t} \tilde{f}_{k}(\mathbf{q}, t)=\frac{g \rho k}{2}\left[\tilde{I}(\mathbf{q})-\frac{2 D_{r} k}{g \rho}\right] \tilde{f}_{k}(\mathbf{q}, t) .
$$

Equation (A12) defines the dispersion relation for the $k$-atic mode dynamics near the disordered state and holds for arbitrary spatial interaction kernels.

\section{Approximation of the pseudodifferential operator and dispersion relation}

In the microscopic model, Eq. (6), we consider an isotropic interaction neighborhood, such that the interaction kernel in Fourier space must be an even function that depends only on the wave-vector amplitude $q=|\mathbf{q}|$. Assuming that $\tilde{I}(x)$ can be expanded in a suitable power series,

$$
\tilde{I}(x)=1+\sum_{j=1}^{\infty} \beta_{j} x^{2 j},
$$

Eq. (A12) has an equivalent interpretation in real space that is given by

$$
\partial_{t} f_{k}(\mathbf{r}, t)=\frac{g \rho k}{2}\left[\tilde{I}\left(R_{\alpha}^{2} \nabla^{2}\right)-\frac{2 D_{r} k}{g \rho}\right] f_{k}(\mathbf{r}, t),
$$

where $\tilde{I}\left(R_{\alpha}^{2} \nabla^{2}\right)$ represents a pseudodifferential operator that is defined by the power series in Eq. (A13).

The equally weighted summation over orientational interactions with particles in an isotropic neighborhood of radius $R_{\alpha}$ corresponds to an interaction kernel $\hat{I}(r)=\Theta\left(r-R_{\alpha}\right)$ in Eq. (A1). The Fourier transform of the appropriately normalized kernel $I(r)=\hat{I}(r) /\left(2 \pi R_{\alpha}^{2}\right)$ defined by Eq. (A3) then reads

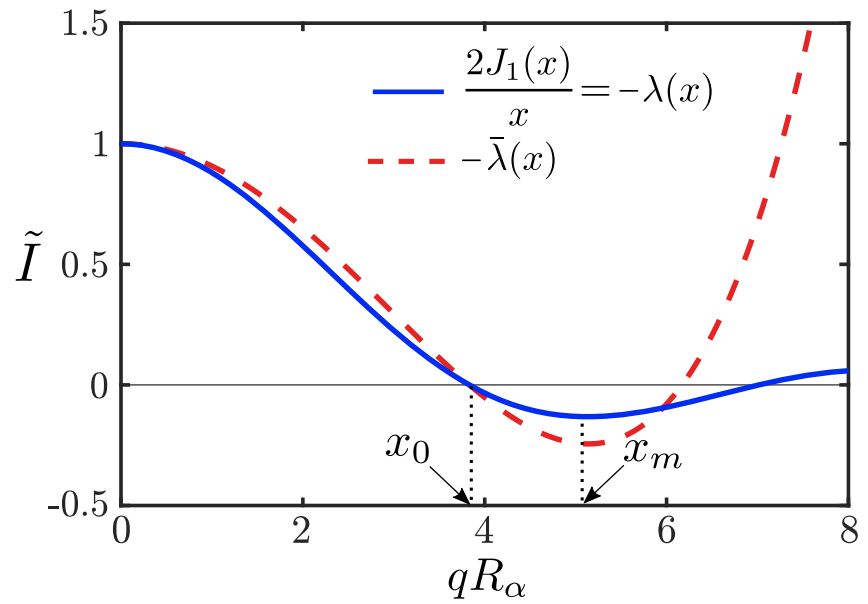

FIG. 9. Fourier-space representation of the interaction kernel $\tilde{I}(x)=-2 J_{1}(x) / x$ (solid line) used in the microscopic particle model, Eq. (6), where $J_{1}$ denotes the Bessel function of the first kind. The linearized mode dynamics, Eq. (A12), implies that, for $D_{r} /(|g| \rho) \ll 1, \lambda=-\tilde{I}(q)$ essentially represents the dispersion relation of the $k$-atic particle model with antialigning interactions $(g<0)$. An empirical approximation $-\bar{\lambda}=1-\beta_{1} x^{2}+\beta_{2} x^{4}$ (dashed line) is then chosen such that the smallest unstable wavelength set by the first root $x_{0}$ and the most unstable wavelength set by $x_{m}$ are the same for $\bar{\lambda}$ and $\lambda$. This yields Eq. (22) with $\beta_{1} \approx 0.1$ and $\beta_{2} \approx 0.002$. 


$$
\tilde{I}(x)=\frac{2 J_{1}(x)}{x}
$$

where $J_{1}$ denotes the Bessel function of the first kind (Fig. 9).

To map the coarse-grained mode dynamics, Eq. (A12), for $k$-atic alignment interactions $(g>0)$ to the GL as a meanfield model, we consider the Taylor series of $\tilde{I}(x)$ given in Eq. (A15) around $x=0$, which implies a nonvanishing coefficient $\beta_{1}=-1 / 8$ in Eq. (A13) and is accurate to $\mathcal{O}\left(x^{4}\right)$. Using this expansion for the operator in Eq. (A14) leads to Eq. (12) and allows for the identification of an effective mean-field correlation length $L$ in terms of the microscopic interaction radius $R_{\alpha}$ [Eq. (13)].

To connect Eq. (A12) to the complex SH equation as a mean-field description of antialigning $k$-atics $(g<0)$, we first note that, for $|g| \rho \gg 1$, we can identify $\lambda(q)=-\tilde{I}(q)$ as the dispersion relation that describes the stability $(\lambda<0)$ or instability $(\lambda>0)$ of homogeneous states under perturbations with wavelength $2 \pi / q$. Consequently, the first interval in which the Fourier-space representation of the interaction kernel (Fig. 9, solid line) changes its sign indicates a band of unstable wave vectors. Because this sign change is not captured by the Taylor series of $\tilde{I}(x)$ up to fourth order around $x=0$, we instead empirically define an approximation $\bar{\lambda}$ such that (i) $\bar{\lambda}(q)=\lambda(-q)$ and $\bar{\lambda}(0)=1$, and (ii) the smallest unstable wave vector and the most unstable wave vector are approximately the same for $\bar{\lambda}(q)$ and $\lambda(q)$ (Fig. 9, dashed line). With this approximation, Eq. (A12) implies the real-space representation Eq. (22) for the linearized mode dynamics and a lengthscale matching as given in Eq. (24).

Finally, we note that a generalization of the hierarchy of mode equations (A6) to the case of finite-range interaction kernels still allows one to decouple the dynamics of modes $n=j k$ for arbitrary integer $j$ from all other modes with $n \neq j k$. However, in this case, the closure assumptions described in Appendix A 1 a lead to additional nonlinear terms in the final dynamic equation of the mode $f_{k}$. For simplicity, these terms, which are $\mathcal{O}\left(f_{k}^{2} \nabla^{2} f_{k}\right)$ to lowest order, have been neglected in Eqs. (12) and (22).

\section{APPENDIX B: LANDAU-DE GENNES (LdG) THEORY OF DLCs WITH $\boldsymbol{k}$-FOLD SYMMETRY}

We first explain how the LdG theory of nematic liquid crystals can be naturally generalized to describe DLCs with arbitrary $k$-fold symmetries. Subsequently, we formally map the resulting relaxation dynamics onto the mean-field equation (1) with $\mathcal{L}=L^{2} \nabla^{2}$ and discuss simple fractional defect solutions in free space. The SH mean-field theory can be discussed in an analogous fashion [83].

\section{Free energy of $\boldsymbol{k}$-atic DLCs in 2D}

Properties of DLCs can be conveniently studied using appropriate free-energy functionals. Typically, these
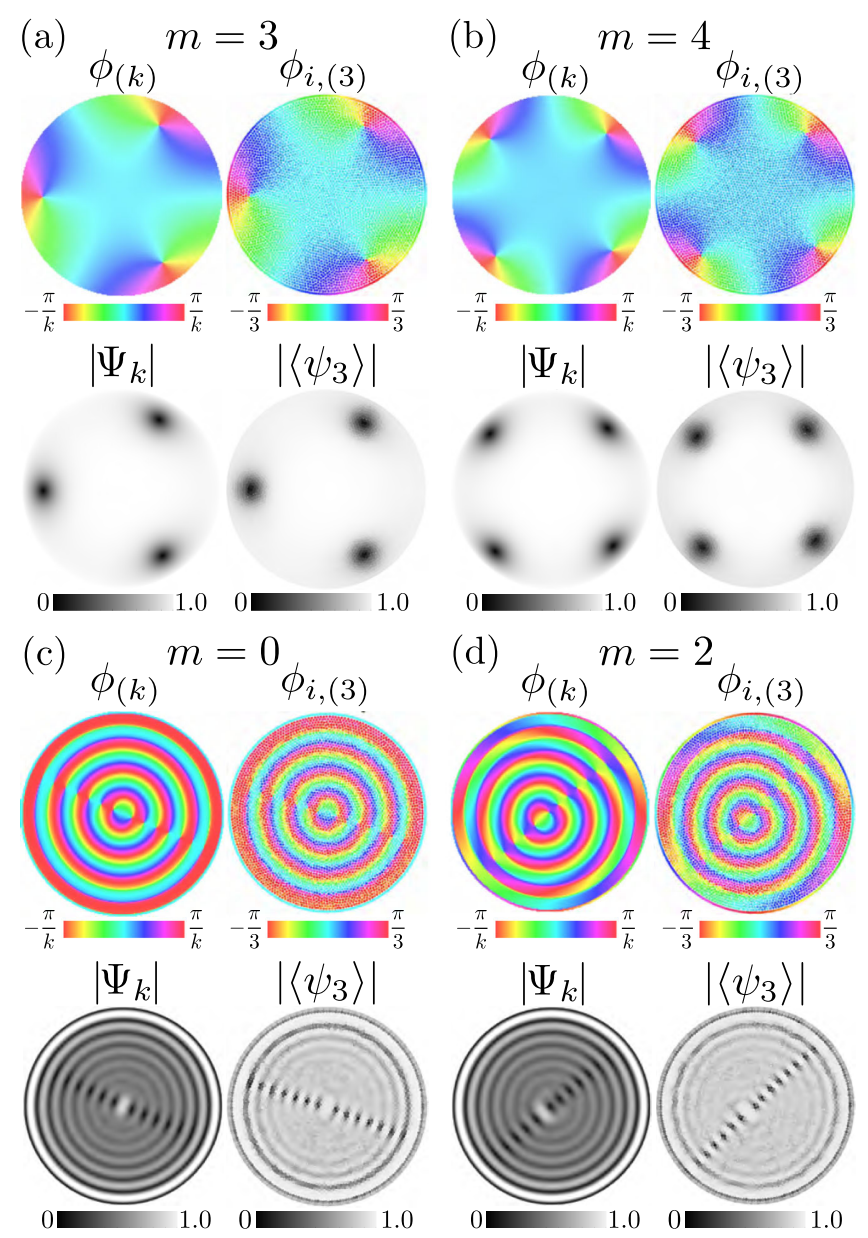

FIG. 10. Additional stationary and long-lived solutions of the "real" GL equation and the particle model, Eq. (6), on a unit disk. (a) Stationary solutions emerging from random initial conditions. Boundary-anchoring equations (14) (left, Eq. (1) with $\mathcal{L}=L^{2} \nabla^{2}$ ) and (17) (right, aligning particle dynamics, Eq. (6), with $k=3$ ) for $m=3$ and boundary-anchoring profile $\gamma_{k}=\theta$ were used. All other parameters are as in Figs. 2(c) and 2(d). (b) Same as (a) for boundary anchoring with $m=4$. (c) Stationary solutions of the complex SH equation emerging from random initial conditions (left) and long-lived solutions in the particle model with antialigning interactions (right) for boundary anchorings with $m=0$. All other parameters are as in Fig. 5(a). Initial director orientations in particle simulations were sampled from the stationary solution of the complex SH equation and evolved according to Eq. (6) until $D_{r} t=500$. (d) Same as (c) for boundary anchoring with $m=2$.

functionals are constructed using vector and tensor-valued fields to encode the underlying microscopic symmetries. Classic examples of this approach are the Frank free energy [73] for polar liquid crystals $(k=1)$ and LdG free energy [1] for nematics $(k=2)$, which are formulated in terms of vectors and traceless symmetric (nematic) tensors, respectively. A particular advantage of this formulation is that a free energy can be systematically constructed as an expansion in terms of the available scalar (rotationally invariant) contractions that can be formed by the given tensorial objects. 
A generalization of this approach to arbitrary $k$-fold symmetric systems can be realized using traceless symmetric tensors of rank $k$, denoted by $Q_{i_{1} \ldots i_{k}}^{(k)}$, which are invariant under rotations of $2 \pi / k$. Note that $k$-atic tensors in two dimensions have only two independent degrees of freedom for any $k$. This is specific to two dimensions, and the total number of independent degrees of freedom increases with $k$ in any higher dimension. In 2D, it is convenient to choose one of these degrees of freedom as

$$
Q_{(k)}=\sqrt{2^{1-k} Q_{i_{1} \ldots i_{k}}^{(k)} Q_{i_{1} \ldots i_{k}}^{(k)}},
$$

which can be identified as the local $k$-atic order. The second degree of freedom can then be chosen as the local orientation of the $k$-atic director $\phi_{(k)} \in(-(\pi / k),(\pi / k)]$. Adopting this parametrization, $k$-atic tensors $Q_{i_{1} \ldots i_{k}}^{(k)}$ are uniquely determined by specifying the two tensor components

$$
\begin{aligned}
& a_{k}:=Q_{x \ldots x x}^{(k)}=Q_{(k)} \cos k \phi_{(k)}, \\
& b_{k}:=Q_{x \ldots x y}^{(k)}=Q_{(k)} \sin k \phi_{(k)},
\end{aligned}
$$

with all other components being implied by the index symmetry and tracelessness. Importantly, the tensor parametrization given in Eqs. (B2) can be used to define the complex $k$-atic order parameter

$$
\Psi_{k}=a_{k}+i b_{k} .
$$

This definition is equivalent to $\Psi_{k}$ given in Eq. (3) and explicitly relates the magnitude $\left|\Psi_{k}\right|=Q_{(k)}$ of $k$-atic order and the $k$-atic phase $\phi_{(k)}$ as introduced in Eq. (4) to a representation of $k$-atic DLCs in terms of traceless symmetric tensors of rank $k$.

To connect a mean-field description of $k$-atic systems in terms of such $k$-atic tensors to Eq. (1), we start from the generic free energy

$$
F_{k}^{\mathrm{GL}}=\int d^{2} r\left[f_{h}+\frac{L^{2}}{2}\left(\partial_{j} Q_{i_{1}, \ldots, i_{k}}^{(k)}\right)^{2}\right],
$$

where $L$ is a parameter describing the $k$-atic system, and homogeneous contributions $f_{h}$ must consist of rotational invariants that can be formed by $k$-atic tensors. In Eq. (B4), the role of $L$ as an effective length scale that penalizes bending of the local $k$-atic director field becomes explicit.

To determine a minimal form of the function $f_{h}$ in Eq. (B4) that is allowed by the underlying symmetries, one has to analyze the possible contractions between $k$-atic tensors that can be constructed to form rotational invariants (scalars). Using the properties of general $k$-atic tensors, one can show that cubic contractions between $k$-atic tensors must vanish for arbitrary $k$-a fact that is well known for nematics $k=2$. Hence, a minimal LdG expansion of $f_{h}$ in the free-energy equation (B4) is, for any $k$, given by

$$
f_{h}=\frac{A}{2} Q_{i_{1} \ldots i_{k}}^{(k)} Q_{i_{1} \ldots i_{k}}^{(k)}+\frac{B}{2^{k+1}}\left(Q_{i_{1} \ldots i_{k}}^{(k)} Q_{i_{1} \ldots i_{k}}^{(k)}\right)^{2}
$$

where $A \in \mathbb{R}$ and $B>0$ are constant material parameters. The relaxation dynamics $\tau \partial_{t} Q_{i_{1} \ldots i_{k}}^{(k)}=-\delta F_{k}^{\mathrm{GL}} / \delta Q_{i_{1} \ldots i_{k}}^{(k)}$ thus takes the form

$\tau \partial_{t} Q_{i_{1} \ldots i_{k}}^{(k)}=-\left(A+B Q_{(k)}^{2}\right) Q_{i_{1} \ldots i_{k}}^{(k)}+L^{2} \nabla^{2} Q_{i_{1} \ldots i_{k}}^{(k)}$,

where $Q_{(k)}$ is defined in Eq. (B1). Using Eqs. (B2) and (B3), we see that the relaxation dynamics, Eq. (B6), is indeed equivalent to mean-field theory of aligning $k$-atic particles, Eq. (1) with $\mathcal{L}=L^{2} \nabla^{2}$, corresponding to a real GL equation for a complex order parameter $\Psi_{k}$.

The latter equivalence also becomes evident on an energetic level through the energy functional $\mathcal{E}_{k}$ given in Eq. (2): For $F_{k}^{\mathrm{GL}}$ given in Eq. (B4), Eqs. (B2) and (B3) imply $\mathcal{E}_{k}=2^{2-k} F_{k}^{\mathrm{GL}}$, and the relaxation dynamics $\tau \partial_{t} \Psi_{k}=$ $-\delta \mathcal{E}_{k} / \delta \Psi_{k}^{*}$ yields the same real GL equation for the complex order parameter $\Psi_{k}$ that we have just identified as being equivalent to Eq. (B6).

\section{Fractional point-defect solutions in free space}

The simplest scenario to study fractional defects in the GL equation, or equivalently in Eq. (B6), is to consider a limit $B=-A \rightarrow \infty$, such that $\left|\Psi_{k}\right|=\sqrt{-A / B}=1$ and the system resides in a perfectly ordered state. Stationary solutions are then determined by

$$
\nabla^{2} \phi_{(k)}=0 .
$$

The regularity of the complex order parameter $\Psi_{k}$ away from the defect demands $k\left[\phi_{(k)}(r, \theta+2 \pi)-\phi_{(k)}(r, \theta)\right]=$ $2 \pi m$ for any integer $m$, where $(r, \theta)$ denote cylindrical coordinates. Hence, physically permissible topological defect solutions of Eq. (B7) can be written as

$$
\phi_{(k), m}(\theta)=\frac{1}{k} \arg \left(e^{i m \theta}\right),
$$

which provides an example for a fractional defect state with topological charge $m / k$ as defined by Eq. (5).

For finite values of $A$ and $B$, the scaling behavior close to and far away from $m / k$ defects can be obtained by following the approach of Ref. [84]. Using an ansatz $\Psi_{k}=$ $Q_{0}(r) \exp \left[i k \phi_{(k)}(\theta)\right]$ in the GL, one again finds stationary fractional $m / k$-defect solutions, Eq. (B8), where the magnitude $Q_{0}(r)$ is now a function of the distance $r$ from the defect 
center. In the $k$-atically ordered regime $A<0$, the magnitude increases near the defect $(r \ll L)$ as $Q_{0} \sim(r / L)^{m}$ and converges far away from the defect $(r \gg L)$ to the value $\sqrt{-A / B}$ with an asymptotic scaling behavior of $Q_{0} \sim 1-(m L)^{2} /\left(2|A| r^{2}\right)$.

\section{APPENDIX C: LANDAU-BRAZOVSKII ENERGY AND SWIFT-HOHENBERG EQUATION}

In this Appendix, we introduce an effective energy that governs the mean-field dynamics of $k$-atic particles with antialigning interactions, the Landau-Brazovskii energy [48], and use it to identify boundary conditions for numerical simulations. We then derive analytic stationary solutions that were used to explain the wavelength doubling and the chiral symmetry breaking, and present additional examples that demonstrate the close agreement between this theory and the particle model.

\section{Free energy and boundary conditions}

The mean-field theory of particles with anti-aligning interactions can be written as relaxation dynamics $\tau \partial_{t} \Psi_{k}=-\delta \mathcal{E}_{k}^{\mathrm{LB}} / \delta \Psi_{k}^{*}$, with energy

$\mathcal{E}_{k}^{\mathrm{LB}}=\int d^{2} r\left(A\left|\Psi_{k}\right|^{2}+\frac{B}{2}\left|\Psi_{k}\right|^{4}-L_{1}^{2}\left|\nabla \Psi_{k}\right|^{2}+L_{2}^{4}\left|\nabla^{2} \Psi_{k}\right|^{2}\right)$,

where $\left|\nabla^{2} \Psi_{k}\right|^{2}=\left(\nabla^{2} \Psi_{k}\right)\left(\nabla^{2} \Psi_{k}^{*}\right)$ and $\mathcal{E}_{k}^{\mathrm{LB}}$ is known as Landau-Brazovskii energy [48]. A general variation of Eq. (C1) with respect to $\Psi^{*}$ yields

$\delta \mathcal{E}_{k}^{\mathrm{LB}}=\int_{S} d^{2} r \delta \Psi_{k}^{*}\left(A+B\left|\Psi_{k}\right|^{2}+L_{1}^{2} \nabla^{2}+L_{2}^{2} \nabla^{2} \nabla^{2}\right) \Psi_{k}+\int_{\partial S} d s \mathbf{n} \cdot\left[\left(L_{1}^{2} \nabla \Psi_{k}-L_{2}^{2} \nabla \nabla^{2} \Psi_{k}\right) \delta \Psi_{k}^{*}+L_{2}^{2}\left(\nabla^{2} \Psi_{k}\right) \nabla \delta \Psi_{k}^{*}\right]$,

where the second term denotes a line integral with boundary normal $\mathbf{n}$ that collects all boundary terms arising from the variation. For the equilibrium condition $\delta \mathcal{E}_{k}^{\mathrm{LB}}=0$, the first integral in Eq. (C2) implies the complex-valued Swift-Hohenberg equation discussed in the main text, Sec. V [Eq. (1) with $\mathcal{L}=-L_{1}^{2} \nabla^{2}-L_{2}^{2} \nabla^{2} \nabla^{2}$ ]. From the condition of vanishing boundary terms in Eq. (C2), suitable boundary conditions can be derived. In particular, we have fixed the order parameter at the boundary through specific anchoring profiles $\left(\Rightarrow \delta \Psi_{k}=0\right)$ and additionally imposed $\left.\nabla^{2} \Psi_{k}\right|_{\partial S}=0$ in numerical simulations (see Appendix D). Therefore, stationary solutions of Eq. (1) with the boundary conditions employed in this work fulfill $\delta \mathcal{E}_{k}^{\mathrm{LB}}=0$ and extremize the energy given in Eq. (C1).

\section{Analytic solutions}

We derive an analytic solution of $\delta \mathcal{E}_{k}^{\mathrm{LB}} / \delta \Psi_{k}^{*}=0$ near the critical transition at $A=A_{*}$ [see Fig. 5(c)]. This solution recapitulates the stationary patterns shown in Fig. 5, including the wavelength doubling between phase and amplitude patterns and the emergence of chiral texture patterns when moving away from the critical point $A_{*}$ (Fig. 6).

For this derivation, we neglect quartic terms proportional to $\left|\Psi_{k}\right|^{4}$ in Eq. (C1) and seek complex order-parameter fields $\Psi_{k}$ that solve

$$
A \Psi_{k}+L_{1}^{2} \nabla^{2} \Psi_{k}+L_{2}^{4} \nabla^{2} \nabla^{2} \Psi_{k}=0 .
$$

We write $A=A_{*}-\Delta A$, where $A_{*}=q_{0}^{4} L_{2}^{4}$ is the critical value of the linear instability and $q_{0}^{2}=L_{1}^{2} /\left(2 L_{2}^{4}\right)$ is the first unstable wave number; see discussion below Eq. (24). With these definitions, via a square completion, Eq. (C3) can be cast into the form

$$
\left(\nabla^{2}+q_{+}^{2}\right)\left(\nabla^{2}+q_{-}^{2}\right) \Psi_{k}=0,
$$

where we have defined $q_{ \pm}^{2}=q_{0}^{2} \pm \sqrt{\Delta A} / L_{2}^{2}$. Equation (C4) represents a bi-Helmholtz equation [85] that can be solved in polar coordinates $(r, \theta)$ by

$$
\Psi_{k}(r, \theta)=\sum_{m=0}^{\infty}\left[\mu_{m} J_{m}\left(q_{-} r\right)+\nu_{m} J_{m}\left(q_{+} r\right)\right] e^{i m \theta},
$$

where $J_{m}(x)$ are Bessel functions of the first kind, and $\mu_{m}$ and $\nu_{m}$ are possibly complex integration constants.

\section{a. Wavelength doubling between amplitude and phase patterns}

At the critical point $A=A_{*}$, we have $q_{-}=q_{+}=q_{0}$, and solutions in Eq. (C5) will be of the form $\Psi_{k} \sim J_{m}\left(q_{0} r\right) e^{i m \theta}$, consistent with the boundary-anchoring equation (14) for $\gamma_{k}=\theta$. Consequently, solutions of this kind contain a topological defect of charge $q_{\mathrm{d}}=m / k$ at $r=0$, and they recapitulate the factor- 2 difference in the wavelength of amplitude patterns $\left|\Psi_{k}\right|$ and phase patterns $\phi_{(k)}$ seen in Fig. 5. To illustrate this for $m=0$ [Figs. 5(a) and 6(a)], we note that the solution $\Psi_{k} \sim J_{0}\left(q_{0} r\right)$ corresponds to

$$
\begin{aligned}
& \left|\Psi_{k}\right| \sim\left|J_{0}\left(q_{0} r\right)\right| \\
& \phi_{(k)}= \begin{cases}0 & \text { for } J_{0}\left(q_{0} r\right) \geq 0 \\
\pi / k & \text { for } J_{0}\left(q_{0} r\right)<0 .\end{cases}
\end{aligned}
$$


As $J_{0}\left(q_{0} r\right)$ oscillates with wavelength $\lambda_{0} \sim 2 \pi / q_{0}$ around zero, Eqs. (C6) imply that phase patterns also have wavelength $\lambda_{0}$, while the amplitude pattern wavelength is $\lambda_{0} / 2$, precisely as observed in numerical simulations [Fig. 5(a)]. Similarly, for $m=1$ [Fig. 6(b)], the solution $\Psi_{k} \sim$ $J_{1}\left(q_{0} r\right) e^{i \theta}$ corresponds to

$$
\begin{aligned}
& \left|\Psi_{k}\right| \sim\left|J_{1}\left(q_{0} r\right)\right| \\
& \phi_{(k)}= \begin{cases}\arg \left(e^{i \theta}\right) / k & \text { for } J_{1}\left(q_{0} r\right) \geq 0 \\
\arg \left(e^{i(\theta-\pi)}\right) / k & \text { for } J_{1}\left(q_{0} r\right)<0,\end{cases}
\end{aligned}
$$

which recapitulates the amplitude and phase patterns shown in the rightmost panel of Fig. 5(b).

\section{b. Analytic solutions with chiral texture patterns}

We derive sufficient conditions for the emergence of chiral texture patterns in the mode $m=1$ of the analytic solution Eq. (C5), denoted in the following as

$$
\Psi_{k}^{(1)}=\left(\mu_{1} J_{-}+\nu_{1} J_{+}\right) e^{i \theta}
$$

with $J_{ \pm}:=J_{1}\left(q_{ \pm} r\right)$. Note that for any choice of complex integration constants $\mu_{1}$ and $\nu_{1}$ in Eq. (C7), the amplitude $\left|\Psi_{k}^{(1)}\right|$ is independent of the polar angle $\theta$ and therefore remains azimuthally symmetric. This is consistent with the various amplitude patterns shown in Fig. 5 of the main text. To determine for which parameters the phase pattern $\phi_{(k)}$ of $\Psi_{k}^{(1)}$ could be chiral, we note that for any complex field $\Psi=|\Psi| e^{i \phi}$, gradients of the phase $\phi$ can be conveniently computed from

$$
\nabla \phi=|\Psi|^{-2} \operatorname{Im}\left(\Psi^{*} \nabla \Psi\right) .
$$

Chirality in texture patterns around defects at $r=0$ can be detected if the radial part of this gradient, $\mathbf{e}_{r} \cdot \nabla \phi$, is different from zero with a fixed sign across the domain, which inspired the phase chirality parameter given in Eq. (26). From Eq. (C8), we find, for $\Psi_{k}^{(1)}$ and $A \leq A_{*}$, the expression

$$
\mathbf{e}_{r} \cdot \nabla \phi_{(k)}=\frac{\operatorname{Im}\left(\mu_{1} \nu_{1}^{*}\right)}{k\left|\Psi_{k}^{(1)}\right|^{2}}\left(J_{+} \partial_{r} J_{-}-J_{-} \partial_{r} J_{+}\right) .
$$

From $\mathbf{e}_{r} \cdot \nabla \phi_{(k)} \neq 0$, we find two necessary conditions from Eq. (C9) for $\Psi_{k}^{(1)}$ given in Eq. (C7) to describe chiral texture patterns: (1) $\mu_{1} \propto \nu_{1}$, such that at least one of the two integration constants must be complex, and (2) $q_{+} \neq q_{-} \Rightarrow A<A_{*}$, meaning that the system has to be in a regime in which a finite band of wave numbers are linearly unstable. The numerical observation that chiral patterns are absent for $A>A_{*}$ additionally constrains the integration constants to $\left|\mu_{1}\right|=\left|\nu_{1}\right|$.

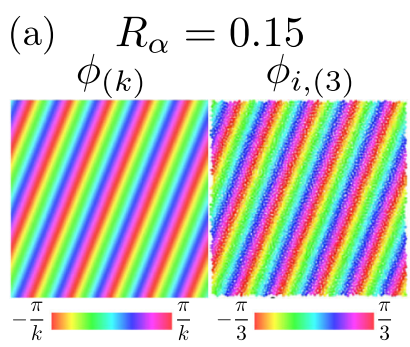

(c) $\quad R_{\alpha}=0.2$
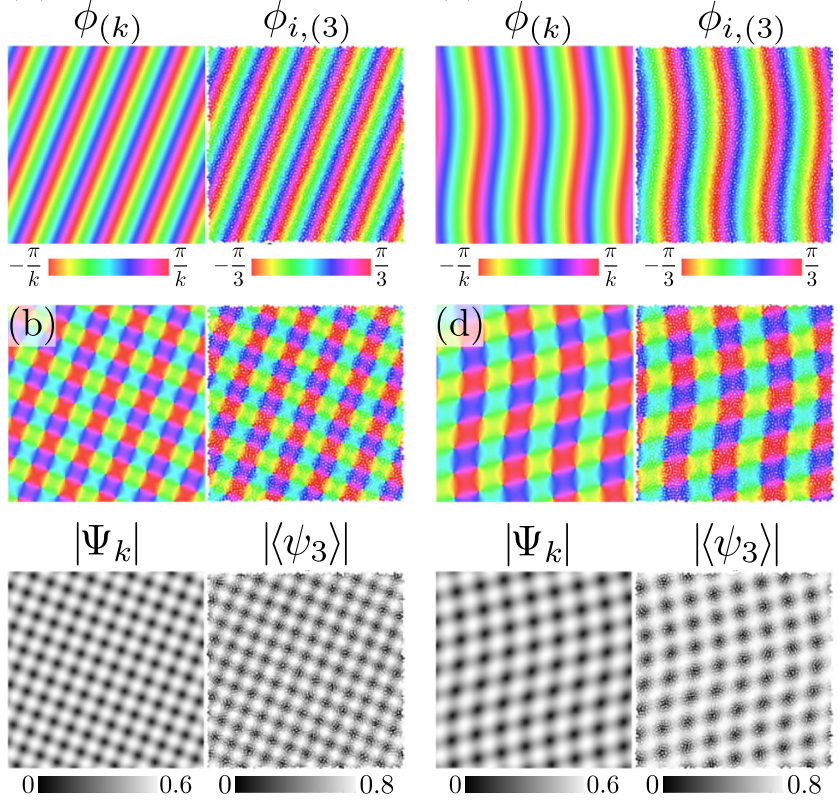

FIG. 11. Spontaneous pattern formation in the SH equation (1) with $\mathcal{L}=-L_{1}^{2} \nabla^{2}-L_{2}^{4}\left(\nabla^{2}\right)^{2}$ and in the particle model with antialignment interactions [Eq. (6) with $g<0 ; 5,000$ particles] on a unit square with periodic boundaries. (a) Example of a defect-free wavelike texture pattern that can be found in both models. (b) Example of a checkerboardlike texture pattern [same color code as in (a)] that contains a $\pm 1 / k$-defect lattice visible in the magnitude of the $k$-atic order parameter $\left|\Psi_{k}\right|$ and in the temporal average $\langle\cdot\rangle$ of the magnitude of the microscopic 3-atic order parameter $\psi_{3}=\sum_{j \in \mathcal{N}_{i}} \exp \left(3 i \alpha_{j}\right) /\left|\mathcal{N}_{i}\right|$. (c,d) Similar patterns for a different microscopic interaction radius and an accordingly modified $\mathrm{SH}$ equation. The phase patterns are reminiscent of those found in polar models of pinwheels in cortical visual maps; see Fig. 4 in Ref. [82]. Parameters: $A=1$, $B=1, L_{1}=0.305 R_{\alpha}$, and $L_{2}=0.205 R_{\alpha}$ (SH equation) and $k=3, g=-1$, and $D_{r}=1$ (particle model).

\section{Patterns on periodic domains}

For completeness, we have additionally studied the antialigning $k$-atic particle model and the complex SH equation on a periodic unit square using numerical simulations (Fig. 11). In both models and for the parameter regime studied in this work, many different texture patterns spontaneously form. These patterns can be broadly grouped in defect-free wavelike [Figs. 11(a) and S4(c)] and checkerboardlike patterns [Figs. 11(b) and S4(d)]. The former have spatially constant order $\left|\Psi_{k}\right|>0$ and are near the critical value $A_{*}=L_{1}^{4} /\left(4 L_{2}^{4}\right)$, essentially given by plane phase waves of the form $\Psi_{k} \sim e^{i \mathbf{q}_{0} \cdot \mathbf{r}}$, with $q_{0}^{2}=L_{1}^{2} /\left(2 L_{2}^{4}\right)$. The latter represent $\pm 1 / k$-defect lattices, as is clearly visible in the order-parameter magnitudes, with vanishing total topological charge. We note that defect-free patterns generally have a lower energy than checkerboard patterns. However, this energy difference is small, and inhomogeneous patterns are generally preferred by the dispersion 
relation $\lambda(q)=-\tilde{I}(q)$ (see Fig. 9, which implies that $q=0$ is stable); this is sufficient for checkerboard patterns to emerge frequently as final stationary textures when starting simulations with random initial conditions. Finally, we find that stationary texture patterns that occur in the complex SH equation are-for all cases that were tested-also stationary in the particle model, if the microscopic directors are initialized with the corresponding $k$-atic phase fields. The opposite is not true: The particle dynamics sometimes gets stuck in long-lived irregular patterns that are not stationary when used in the SH equation.

\section{APPENDIX D: NUMERICAL SIMULATIONS}

In the following, we discuss the coefficient matching and comparison of relaxation timescales between the meanfield model and microscopic simulations. Finally, we summarize details of the different methods and workflows that have been used to generate the numerical results presented in this work.

\section{Matching homogeneous mean-field coefficients with parameters of the microscopic model}

To match the homogeneous coefficients $A$ and $B$ in Eq. (1) to the microscopic dynamics, we note that the latter was considered in a regime of high particle density, viz. $D_{r} /(|g| \rho) \ll 1$. In this case, Eq. (11) implies $\bar{A} \approx-1$ for aligning interactions $(g>0)$ and $\bar{A} \approx 1$ for antialigning interactions $(g<0)$. Accordingly, throughout this work, we have set $A=-1$ for simulations of the GL equation [Eq. (1) with $\mathcal{L}=L^{2} \nabla^{2}$ ] and $A=1$ for simulations of the SH equation [Eq. (1) with $\mathcal{L}=-L_{1}^{2} \nabla^{2}-L_{2}^{4}\left(\nabla^{2}\right)^{2}$ ]. The coefficient $B$ was set empirically: Away from defects, we expect $\left|\Psi_{k}\right| \simeq\left|\psi_{k}\right| \approx 1$ for an ordered state in the microscopic model, which is ensured in the GL equation by setting $B=1$. The same value is adopted in simulations of the SH equation, where it also leads to good agreement with order-parameter magnitudes of the microscopic model with $g<0$.

\section{Comparison of characteristic timescales}

The relaxation timescale introduced with the generalized GL equation (1) is given by $\tau$. From the coarse-graining result, Eq. (10), we expect $\tau$ to be comparable to the timescale $\bar{\tau}=2 /(|g| k \rho)$ if the mean-field parameters and operators $\mathcal{L}$ are matched according to Eqs. (11), (13), and (24). To test this, we have to compare observations from a dynamic process as described by the generalized GL equation (1) and by the microscopic model, Eq. (6). To this end, we refer to the defect relaxation dynamics depicted in Figs. 2(c) and 2(d), where the time points of snapshots of the "real" GL equation simulation in units of $\tau$ are provided in the caption. The timescale of particle simulations was set, for practical reasons, by the inverse rotational diffusion constant $1 / D_{r}$; i.e., in units of $\bar{\tau}$, it was given by $s \bar{\tau}$ with

$$
s=\frac{2 D_{r}}{|g| k \rho} .
$$

Scaling numerical time points of particle simulations for the given parameters accordingly $(g=0.25, k=3$, $\rho=4000 / \pi, D_{r}=1$ ) then leads to the temporal coordinates $t / \bar{\tau}$ of the particle model snapshots listed in the caption of Fig. 2(d). These snapshots were chosen such that they best resemble textures from the mean-field model. From the relative values of corresponding time points, we can estimate $\bar{\tau} / \tau \approx 0.4$, indicating that the coarse graining predicts a slightly faster relaxation dynamics than actually exhibited by the matched mean-field model, Eq. (1).

\section{Numerical methods: Mean-field simulations}

Real and imaginary parts of the GL equation (1) with $\mathcal{L}=$ $L^{2} \nabla^{2}$ were simulated separately on the unit disk using the finite element partial differential equation solver provided by MATLAB [86]. Boundary-anchoring profiles described in Eq. (14) were imposed as Dirichlet boundary conditions.

For the case of the $\mathrm{SH}$ equation, Eq. (1) with $\mathcal{L}=$ $-L_{1}^{2} \nabla^{2}-L_{2}^{4}\left(\nabla^{2}\right)^{2}$ was rewritten as a system of two pairs of second-order differential equations, and $\left.\nabla^{2} \Psi_{k}\right|_{\partial S}=0$ was included as an additional boundary condition (see Appendix C 1). The open-source Dedalus framework [87] was used to spectrally solve the SH equation on a periodic domain with $256 \times 256$ grid points using a Fourier basis and integration time steps of $d t=10^{-2}$ in units of $\tau$.

\section{Numerical methods: Microscopic model}

Bulk particles were first randomly positioned on the respective domain (a unit disk or a square with periodic boundary). For simulations on the unit disk, a single line of boundary particles with fixed positions was additionally placed along the outline of the disk. In the next step, the bulk particles were left to distribute themselves homogeneously in space in the absence of noise via a pairwise repulsive force $\mathbf{f} \sim \nabla \exp \left(-r_{p}^{2} / L_{f}^{2}\right)$, where $L_{f}^{2}=A / N$ for $N$ particles distributed on a domain of area $A$. After that, all particle positions were kept fixed, and Eq. (6) was integrated using the Euler-Maruyama method [88] with integration time steps of $d t=10^{-4}$ in units of $1 / D_{r}$. To realize the boundary anchoring on the unit disk, boundary particles did not participate in the stochastic dynamics but kept the fixed director angle profile given in Eq. (17) and acted as neighbors for the director dynamics of bulk particles.

\section{Initial conditions}

Random initial conditions have been used for most of the simulation results shown, except for the time series in 
Figs. 2(c) and 2(d), as well as to generate the steady-state pattern in Fig. 5(a) $(m=0)$ and the initial state $t=0$ in Fig. 7 $(m=2)$. To describe the initial conditions for the latter cases, in the following, we denote $(r, \theta)$ and $\left(r_{i}, \theta_{i}\right)$ as the position of a given field or particle position in cylindrical coordinates. The initial point-defect states in Figs. 2(c) and 2(d) $(t=0)$ are, respectively, given by $\Psi_{k}=e^{i 2 \theta}$ and $\alpha_{i}=(2 / 3) \theta_{i}$ ( $k=3$ in the particle model), which maps to the $k$-atic phase angles $\phi_{(k)}$ and $\phi_{(k), i}$ as shown in Eqs. (15) and (16). To generate spiral stationary states that do not form spontaneously, we use $h_{m}(r, \theta)=8 \pi(r-1)+m \theta$ and initialize the order-parameter field as $\Psi_{k}=e^{i h_{m}(r, \theta)}$ and the particle director field as $\alpha_{i}=\left[h_{m}\left(r_{i}, \theta_{i}\right) / 3 \bmod 2 \pi\right](k=3$ in the particle model). The equilibration of these states gives rise to the patterns shown in Fig. 5(a) $(m=0)$ and to the initial state $t=0$ in Fig. $7(m=2)$.

[1] P. G. de Gennes and J. Prost, The Physics of Liquid Crystals (Clarendon Press, Oxford, 1993).

[2] P. M. Chaikin and T. C. Lubensky, Principles of Condensed Matter Physics (Cambridge University Press, Cambridge, England, 2000).

[3] Q. Liu, P. J. Ackerman, T. C. Lubensky, and I. I. Smalyukh, Biaxial Ferromagnetic Liquid Crystal Colloids, Proc. Natl. Acad. Sci. U.S.A. 113, 10479 (2016).

[4] M. Niederberger, Multiscale Nanoparticle Assembly: From Particulate Precise Manufacturing to Colloidal Processing, Adv. Funct. Mater. 27, 1703647 (2017).

[5] M. Fruchart, Y. Zhou, and V. Vitelli, Dualities and NonAbelian Mechanics, Nature (London) 577, 636 (2020).

[6] Z. L. Wang and X. Feng, Polyhedral Shapes of $\mathrm{CeO}_{2}$ Nanoparticles, J. Phys. Chem. B 107, 13563 (2003).

[7] H. Dong, S.-R. Du, X.-Y. Zheng, G.-M. Lyu, L.-D. Sun, L.-D. Li, P.-Z. Zhang, C. Zhang, and C.-H. Yan, Lanthanide Nanoparticles: From Design toward Bioimaging and Therapy, Chem. Rev. 115, 10725 (2015).

[8] K. Zhao, R. Bruinsma, and T. G. Mason, Local Chiral Symmetry Breaking in Triatic Liquid Crystals, Nat. Commun. 3, 801 (2012).

[9] G.-R. Yi, D. J. Pine, and S. Sacanna, Recent Progress on Patchy Colloids and Their Self-Assembly, J. Phys. Condens. Matter 25, 193101 (2013).

[10] H. R. Vutukuri, A. Imhof, and A. van Blaaderen, Fabrication of Polyhedral Particles from Spherical Colloids and Their Self-Assembly into Rotator Phases, Angew. Chem. Int. Ed. 53, 13830 (2014).

[11] M. Sindoro, N. Yanai, A.-Y. Jee, and S. Granick, ColloidalSized Metal-Organic Frameworks: Synthesis and Applications, Acc. Chem. Res. 47, 459 (2014).

[12] B. Li, D. Zhou, and Y. Han, Assembly and Phase Transitions of Colloidal Crystals, Nat. Rev. Mater. 1, 1 (2016).

[13] C. Avci, I. Imaz, A. Carné-Sánchez, J. A. Pariente, N. Tasios, J. Pérez-Carvajal, M. I. Alonso, A. Blanco, M. Dijkstra, C. López, and D. Maspoch, Self-Assembly of Polyhedral Metal-Organic Framework Particles into
Three-Dimensional Ordered Superstructures, Nat. Chem. 10, 78 (2018).

[14] R. C. Löffler, Master's thesis, Universität Konstanz, 2018.

[15] Y. Wang, Y. Wang, X. Zheng, G.-R. Yi, S. Sacanna, D. J. Pine, and M. Weck, Three-Dimensional Lock and Key Colloids, J. Am. Chem. Soc. 136, 6866 (2014).

[16] A.-C. Genix and J. Oberdisse, Nanoparticle Self-Assembly: From Interactions in Suspension to Polymer Nanocomposites, Soft Matter 14, 5161 (2018).

[17] K. Zhao and T. G. Mason, Assembly of Colloidal Particles in Solution, Rep. Prog. Phys. 81, 126601 (2018).

[18] L. Giomi, Geometry and Topology of Turbulence in Active Nematics, Phys. Rev. X 5, 031003 (2015).

[19] A. P. Gantapara, W. Qi, and M. Dijkstra, A Novel Chiral Phase of Achiral Hard Triangles and an Entropy-Driven Demixing of Enantiomers, Soft Matter 11, 8684 (2015).

[20] S. Dussi and M. Dijkstra, Entropy-Driven Formation of Chiral Nematic Phases by Computer Simulations, Nat. Commun. 7, 11175 (2016).

[21] J. A. Anderson, J. Antonaglia, J. A. Millan, M. Engel, and S. C. Glotzer, Shape and Symmetry Determine Two-Dimensional Melting Transitions of Hard Regular Polygons, Phys. Rev. X 7, 021001 (2017).

[22] M. J. Bowick, O. V. Manyuhina, and F. Serafin, Shapes and Singularities in Triatic Liquid-Crystal Vesicles, Europhys. Lett. 117, 26001 (2017).

[23] A. J. Beekman, J. Nissinen, K. Wu, K. Liu, R.-J. Slager, Z. Nussinov, V. Cvetkovic, and J. Zaanen, Dual Gauge Field Theory of Quantum Liquid Crystals in Two Dimensions, Phys. Rep. 683, 1 (2017).

[24] P. Sartori and C. F. Lee, Scaling Behaviour of Nonequilibrium Planar n-atic Spin Systems under Weak Fluctuations, New J. Phys. 21, 073064 (2019).

[25] A. Maitra, M. Lenz, and R. Voituriez, Chiral Active Hexatics: Giant Number Fluctuations, Waves, and Destruction of Order, Phys. Rev. Lett. 125, 238005 (2020).

[26] F. Vafa, Defect Dynamics in Active Polar Fluids vs. Active Nematics, arXiv:2009.10723.

[27] F. Vafa, M. J. Bowick, M. C. Marchetti, and B. I. Shraiman, Multi-Defect Dynamics in Active Nematics, arXiv: 2007.02947.

[28] L. Giomi, J. Toner, and N. Sarkar, Hydrodynamic Theory of p-atic Liquid Crystals, arXiv:2106.11957.

[29] P. G. de Gennes, An Analogy between Superconductors and Smectics A, Solid State Commun. 10, 753 (1972).

[30] B. I. Halperin and T. C. Lubensky, On the Analogy between Smectic a Liquid Crystals and Superconductors, Solid State Commun. 14, 997 (1974).

[31] B. I. Halperin, T. C. Lubensky, and S. Ma, First-Order Phase Transitions in Superconductors and Smectic-a Liquid Crystals, Phys. Rev. Lett. 32, 292 (1974).

[32] P. S. Pershan and J. Prost, Landau Theory of the Reentrant Nematic-Smectic A Phase Transition, J. Phys. Lett. 40, 27 (1979).

[33] S. R. Renn and T. C. Lubensky, Abrikosov Dislocation Lattice in a Model of the Cholesteric-to-Smectic-A Transition, Phys. Rev. A 38, 2132 (1988).

[34] M. J. Bowick and L. Giomi, Two-Dimensional Matter: Order, Curvature and Defects, Adv. Phys. 58, 449 (2009). 
[35] B. Loewe, A. Souslov, and P. M. Goldbart, Flocking from a Quantum Analogy: Spin-Orbit Coupling in an Active Fluid, New J. Phys. 20, 013020 (2018).

[36] A. Souslov, B. Loewe, and P. M. Goldbart, Emergent Tilt Order in Dirac Polymer Liquids, Phys. Rev. E 92, 030601 (R) (2015).

[37] B. Zappone, A. E. Mamuk, I. Gryn, V. Arima, A. Zizzari, R. Bartolino, E. Lacaze, and R. Petschek, Analogy between Periodic Patterns in Thin Smectic Liquid Crystal Films and the Intermediate State of Superconductors, Proc. Natl. Acad. Sci. U.S.A. 117, 17643 (2020).

[38] F. Wilczek, Quantum Mechanics of Fractional-Spin Particles, Phys. Rev. Lett. 49, 957 (1982).

[39] S. R. Lee, P. A. Sharma, A. L. Lima-Sharma, W. Pan, and T. M. Nenoff, Topological Quantum Materials for Realizing Majorana Quasiparticles, Chem. Mater. 31, 26 (2019).

[40] J. M. Leinaas and J. Myrheim, On the Theory of Identical Particles, Il Nuovo Cimento B 37, 1 (1977).

[41] R. B. Laughlin, Anomalous Quantum Hall Effect: An Incompressible Quantum Fluid with Fractionally Charged Excitations, Phys. Rev. Lett. 50, 1395 (1983).

[42] D. Arovas, J. R. Schrieffer, and F. Wilczek, Fractional Statistics and the Quantum Hall Effect, Phys. Rev. Lett. 53, 722 (1984).

[43] B. I. Halperin, The Quantized Hall Effect, Sci. Am. 254, 52 (1986).

[44] J. Nakamura, S. Fallahi, H. Sahasrabudhe, R. Rahman, S. Liang, G. C. Gardner, and M. J. Manfra, Aharonov-Bohm Interference of Fractional Quantum Hall Edge Modes, Nat. Phys. 15, 563 (2019).

[45] H. Bartolomei, M. Kumar, R. Bisognin, A. Marguerite, J.-M. Berroir, E. Bocquillon, B. Plaçais, A. Cavanna, Q. Dong, U. Gennser, Y. Jin, and G. Fève, Fractional Statistics in Anyon Collisions, Science 368, 173 (2020).

[46] A. Kitaev, Fault-Tolerant Quantum Computation by Anyons, Ann. Phys. (Amsterdam) 303, 2 (2003).

[47] C. Nayak, S. H. Simon, A. Stern, M. Freedman, and S. D. Sarma, Non-Abelian Anyons and Topological Quantum Computation, Rev. Mod. Phys. 80, 1083 (2008).

[48] S. A. Brazovskii, Phase Transition of an Isotropic System to a Nonuniform State, Sov. Phys. JETP 41, 85 (1975).

[49] J. Chao, H. Zhang, Y. Xing, Q. Li, H. Liu, L. Wang, L. Wang, and C. Fan, Programming DNA Origami Assembly for Shape-Resolved Nanomechanical Imaging Labels, Nat. Protoc. 13, 1569 (2018).

[50] R. Veneziano, S. Ratanalert, K. Zhang, F. Zhang, H. Yan, W. Chiu, and M. Bathe, Designer Nanoscale DNA Assemblies Programmed from the Top Down, Science 352, 1534 (2016).

[51] M. Siavashpouri, C. H. Wachauf, M. J. Zakhary, F. Praetorius, H. Dietz, and Z. Dogic, Molecular Engineering of Chiral Colloidal Liquid Crystals Using DNA Origami, Nat. Mater. 16, 849 (2017).

[52] V. Soni, E. S. Bililign, S. Magkiriadou, S. Sacanna, D. Bartolo, M. J. Shelley, and W. T. M. Irvine, The Odd Free Surface Flows of a Colloidal Chiral Fluid, Nat. Phys. 15, 1188 (2019).

[53] E. W. Gehrels, W. B. Rogers, and V. N. Manoharan, Using DNA Strand Displacement to Control Interactions in DNA-Grafted Colloids, Soft Matter 14, 969 (2018).
[54] I. S. Aranson and L. Kramer, The World of the Complex Ginzburg-Landau Equation, Rev. Mod. Phys. 74, 99 (2002).

[55] M. Cross and H. Greenside, Pattern Formation and Dynamics in Nonequilibrium Systems (Cambridge University Press, Cambridge, England, 2009).

[56] E. P. Gross, Structure of a Quantized Vortex in Boson Systems, Nuovo Cimento (1955-1965) 20, 454 (1961).

[57] V. Heinonen, K. J. Burns, and J. Dunkel, Quantum Hydrodynamics for Supersolid Crystals and Quasicrystals, Phys. Rev. A 99, 063621 (2019).

[58] F. S. Bates, J. H. Rosedale, and G. H. Fredrickson, Fluctuation Effects in a Symmetric Diblock Copolymer near the Order-Disorder Transition, J. Chem. Phys. 92, 6255 (1990).

[59] R. K. W. Spencer and R. A. Wickham, Simulation of Nucleation Dynamics at the Cylinder-to-Lamellar Transition in a Diblock Copolymer Melt, Soft Matter 9, 3373 (2013).

[60] A. Ciach, J. Pękalski, and W. T. Góźdź, Origin of Similarity of Phase Diagrams in Amphiphilic and Colloidal Systems with Competing Interactions, Soft Matter 9, 6301 (2013).

[61] L. N. Carenza, G. Gonnella, A. Lamura, D. Marenduzzo, G. Negro, and A. Tiribocchi, Soft Channel Formation and Symmetry Breaking in Exotic Active Emulsions, Sci. Rep. 10, 15936 (2020).

[62] J. Swift and P. C. Hohenberg, Hydrodynamic Fluctuations at the Convective Instability, Phys. Rev. A 15, 319 (1977).

[63] P. C. Hohenberg and B. I. Halperin, Theory of Dynamic Critical Phenomena, Rev. Mod. Phys. 49, 435 (1977).

[64] M. Janoschek, M. Garst, A. Bauer, P. Krautscheid, R. Georgii, P. Böni, and C. Pfleiderer, Fluctuation-Induced First-Order Phase Transition in Dzyaloshinskii-Moriya Helimagnets, Phys. Rev. B 87, 134407 (2013).

[65] J. M. Kosterlitz and D. J. Thouless, Ordering, Metastability and Phase Transitions in Two-Dimensional Systems, J. Phys. Condens. Matter 6, 1181 (1973).

[66] J. McCarthy, Numerical Simulation of the XY-Model on a Two-Dimensional Random Lattice, Nucl. Phys. B275, 421 (1986).

[67] D. S. Dean, Langevin Equation for the Density of a System of Interacting Langevin Processes, J. Phys. A 29, L613 (1996).

[68] E. Bertin, M. Droz, and G. Grégoire, Hydrodynamic Equations for Self-Propelled Particles: Microscopic Derivation and Stability Analysis, J. Phys. A 42, 445001 (2009).

[69] F. D. C. Farrell, M. C. Marchetti, D. Marenduzzo, and J. Tailleur, Pattern Formation in Self-Propelled Particles with Density-Dependent Motility, Phys. Rev. Lett. 108, 248101 (2012).

[70] R. Großmann, P. Romanczuk, M. Bär, and L. SchimanskyGeier, Vortex Arrays and Mesoscale Turbulence of SelfPropelled Particles, Phys. Rev. Lett. 113, 258104 (2014).

[71] K. H. Nagai, Y. Sumino, R. Montagne, I. S. Aranson, and H. Chaté, Collective Motion of Self-Propelled Particles with Memory, Phys. Rev. Lett. 114, 168001 (2015).

[72] D. Arold and M. Schmiedeberg, Mean Field Approach of Dynamical Pattern Formation in Underdamped Active Matter with Short-Ranged Alignment and Distant Antialignment Interactions, J. Phys. Condens. Matter 32, 315403 (2020). 
[73] S. Chandrasekhar, Liquid Crystals (Cambridge University Press, Cambridge, England, 1992).

[74] J. Pollard, G. Posnjak, S. Čopar, I. Muševič, and G. P. Alexander, Point Defects, Topological Chirality, and Singularity Theory in Cholesteric Liquid-Crystal Droplets, Phys. Rev. X 9, 021004 (2019).

[75] L. Tran and K. J. M. Bishop, Swelling Cholesteric Liquid Crystal Shells to Direct the Assembly of Particles at the Interface, ACS Nano 14, 5459 (2020).

[76] B. A. Grzybowski, H. A. Stone, and G. M. Whitesides, Dynamic Self-Assembly of Magnetized, MillimetreSized Objects Rotating at a Liquid-Air Interface, Nature (London) 405, 1033 (2000).

[77] A. Walther and A. H. E. Müller, Janus Particles: Synthesis, Self-Assembly, Physical Properties, and Applications, Chem. Rev. 113, 5194 (2013).

[78] H. H. Wensink, H. Löwen, M. Marechal, A. Härtel, R. Wittkowski, U. Zimmermann, A. Kaiser, and A. M. Menzel, Differently Shaped Hard Body Colloids in Confinement: From Passive to Active Particles, Eur. Phys. J. ST 222, 3023 (2013).

[79] R. Alert, J.-F. Joanny, and J. Casademunt, Universal Scaling of Active Nematic Turbulence, Nat. Phys. 16, 682 (2020).

[80] E. Bertin, A. Baskaran, H. Chaté, and M. C. Marchetti, Comparison between Smoluchowski and Boltzmann Approaches for Self-Propelled Rods, Phys. Rev. E 92, 042141 (2015).
[81] B. Liebchen, M. E. Cates, and D. Marenduzzo, Pattern Formation in Chemically Interacting Active Rotors with Self-Propulsion, Soft Matter 12, 7259 (2016).

[82] H. Y. Lee, M. Yahyanejad, and M. Kardar, Symmetry Considerations and Development of Pinwheels in Visual Maps, Proc. Natl. Acad. Sci. U.S.A. 100, 16036 (2003).

[83] A. U. Oza and J. Dunkel, Antipolar Ordering of Topological Defects in Active Liquid Crystals, New J. Phys. 18, 093006 (2016).

[84] E. Bodenschatz, W. Pesch, and L. Kramer, Structure and Dynamics of Dislocations in an Anisotropic PatternForming Systems, Physica (Amsterdam) 32D, 135 (1988).

[85] T. Askham, A Stabilized Separation of Variables Method for the Modified Biharmonic Equation, J. Sci. Comput. 76, 1674 (2018).

[86] MATLAB 2019b, the MathWorks, Natick, MA, USA.

[87] K. J. Burns, G. M. Vasil, J. S. Oishi, D. Lecoanet, and B. P. Brown, Dedalus: A Flexible Framework for Numerical Simulations with Spectral Methods, Phys. Rev. Research 2, 023068 (2020).

[88] P. E. Kloeden and E. Platen, Numerical Solution of Stochastic Differential Equations (Springer, Berlin, Heidelberg, 2011).

[89] See Supplemental Material at http://link.aps.org/ supplemental/10.1103/PhysRevX.12.011027 for numerical simulations of particle model and mean-field theory demonstrating defect formation, defect braiding, and chiral symmetry-breaking (Movies 1-8). 